\title{
The weak substitution method - An application of the mortar method for patch coupling in NURBS-based isogeometric analysis
}

\author{
W. Dornisch*, G. Vitucci, S. Klinkel \\ Lehrstuhl für Baustatik und Baudynamik, RWTH Aachen, Mies-van-der-Rohe-Str. 1, 52074 Aachen, Germany
}

\begin{abstract}
In this contribution a mortar-type method for the coupling of non-conforming NURBS surface patches is proposed. The connection of non-conforming patches with shared degrees of freedom requires mutual refinement, which propagates throughout the whole patch due to the tensor-product structure of NURBS surfaces. Thus, methods to handle non-conforming meshes are essential in NURBS-based isogeometric analysis. The main objective of this work is to provide a simple and efficient way to couple the individual patches of complex geometrical models without altering the variational formulation. The deformations of the interface control points of adjacent patches are interrelated with a master-slave relation. This relation is established numerically using the weak form of the equality of mutual deformations along the interface. With the help of this relation the interface degrees of freedom of the slave patch can be condensated out of the system. A natural connection of the patches is attained without additional terms in the weak form. The proposed method is also applicable for nonlinear computations without further measures. Linear and geometrical nonlinear examples show the high accuracy and robustness of the new method. A comparison to reference results and to computations with the Lagrange multiplier method is given.
\end{abstract}

Keywords: Nonlinear isogeometric analysis, NURBS, Domain decomposition, Weak substitution method, Multi-patch connection, Mortar methods

\section{Introduction}

Isogeometric analysis was introduced in 2005 by Hughes et al. [1]. The main paradigm is to use the geometry description and the basis functions of the design model also for the analysis. Thus, the geometry is represented exactly on element level. Furthermore, the usage of one common geometry model simplifies the realization of an integrated design and analysis work flow. Several different geometry descriptions are subject of current research in isogeometric analysis. NURBS [1, 2, 3], T-splines [4] and subdivision surfaces [5] are the most common ones. Akin to the prevalence of NURBS surfaces in industrial design, currently NURBS have the biggest share in isogeometric research. However, NURBS surfaces have two prominent drawbacks which impede the use of NURBS elements for isogeometric analysis:

- Complex geometries usually consist of several patches, which are not connected to each other in the geometry model. The discretization of adjacent patches is generally non-conforming.

- The tensor-product structure of NURBS surfaces precludes local refinement. Refinement always propagates throughout the entire patch.

${ }^{*}$ Correspondence to: W. Dornisch, Lehrstuhl für Technische Mechanik, Technische Universität Kaiserslautern, Gottlieb-Daimler-Str., 67663 Kaiserslautern, Germany. E-mail: dornisch@rhrk.uni-kl.de

This is the peer reviewed version of the following article: Dornisch, W, Vitucci, G, and Klinkel, S (2015): The weak substitution method - an application of the mortar method for patch coupling in NURBS-based isogeometric analysis. Int. J. Numer. Meth. Engng, 103, 205-234, which has been published in final form at doi: 10.1002/nme.4918. This article may be used for noncommercial purposes in accordance with Wiley Terms and Conditions for Self-Archiving. 
The combination of these two issues requires methods to handle non-conforming patches in order to establish an efficient mechanical response analysis framework for the computation of complex NURBS surface models.

Domain decomposition methods decompose the domain of a given problem into subdomains, of which the parametrization does not have to match. The situation at hand is an ideal field of application for domain decomposition methods, where each patch can be regarded as one subdomain. A general overview over domain decomposition methods is given in [6]. The Penalty method [7], the Lagrange multiplier method [8] and Nitsche's method [9] were proposed in the 1970s to enforce Dirichlet boundary conditions in a weak manner. In recent engineering literature, these methods are used as a basis for domain decomposition techniques. Mortar methods are the prevailing domain decomposition method in mathematical literature. Basically, two standard types of mortar formulations exist, which are referred to as the non-conforming positive definite problem and as the saddle point problem based on unconstrained product spaces in [6]. In all cases a Lagrange multiplier space is used for the matching conditions along the interface. The mortar method leading to the non-conforming positive definite problem was initially proposed by Bernardi et al. [10]. The function spaces are constrained to fulfill the matching condition at the interface in a weak manner. Basically, this means that the basis functions of one side are related to those of the other side in a way, that the matching condition is fulfilled. The discretization of the interface condition with Lagrange multiplier functions yields an equation which contains a mass matrix of the basis functions. The inversion of this matrix allows the constrained basis functions to be computed. The Lagrange multiplier is not contained in the variational formulation, which remains positive definite. The second mortar approach, which leads to the saddle point problem based on unconstrained product spaces, was proposed by Ben Belgacem [11]. In this case the interface conditions are not enforced with the help of constrained function spaces, but directly realized by means of Lagrange multipliers. This approach enhances the variational formulation by Lagrange multiplier terms to enforce the interface condition in a weak manner. It is basically equal to engineering formulations basing on the Lagrange multiplier method [8]. The variational formulation turns into a saddle point problem. Commonly finite element trace spaces are used for the Lagrange multiplier. This yields non-local basis functions on one side of the interface for the non-conforming positive definite problem, which is the method of choice in this work. The usage of dual basis spaces for the Lagrange multiplier is proposed by Wohlmuth in $[6,12]$. The biorthogonality of the dual spaces yields local support of the basis function. Thus, this choice potentially leads to higher numerical efficiency. A method to compute dual basis spaces for polynomials of arbitary order is presented in [13].

In the framework of isogeometric analysis the following works focus on the domain coupling of NURBS patches. Hesch and Betsch [14] proposed a finite element mortar method for the domain decomposition of transient large deformation problems, and adapted this formulation to isogeometric analysis in [15]. Both formulations use a Lagrange multiplier field to add the work done by coupling tractions along the interface to the weak form. The Lagrange multiplier field is discretized with linear Lagrange basis functions. Mortar integrals are used to establish discrete mesh tying constraints, which constrain the deformations of adjacent faces to be equal within the time discretization. Brivadis et al. [16] presented an isogeometric mortar method, where the weak form is enhanced by Lagrange multiplier terms in order to enforce continuity across the interface. Several choices of Lagrange multiplier spaces are investigated. Basing on [13], a possibility to construct biorthogonal spaces for the Lagrange multiplier space is sketched, but not followed further. This choice would entail compact support of the basis functions, which is otherwise not the case. Apostolatos et al. [17] applied domain decomposition methods basing on the Penalty method, the Lagrange multiplier method, the augmented Lagrange method and Nitsche's method to 2D linear elasticity. These four methods are compared with the help of several benchmark examples. The work of Ruess et al. [18] uses Nitsche's method in combination with an immersed boundary method to model complex geometries described by trimmed NURBS. The work of Nguyen at al. [19] also uses Nitsche's method for the coupling of domains.

All methods mentioned above have in common that the variational formulation is enriched with additional terms. Additional variable fields are introduced and thus the formulation gets more complex. Additional matrices have to be computed. In the case of the Penalty method an empirical parameter has to be determined, which influences the quality of the fulfillment of the constraints and the impact on the condition of the stiffness matrix. The Lagrange multiplier method as well as the mortar method according to Ben Belgacem [11] introduce Lagrange parameters as additional global degrees of freedom. The choice of the function spaces for these parameters influences the results. The condition of the stiffness matrix usually deteriorates if no appropriate pre-conditioner is applied. Nitsche's method neither requires an empirical parameter nor additional degrees of freedom. But the effort for implementation is comparatively high and the solution costs are higher, as an additional eigenvalue problem has to be solved, see [17]. 
The connection of hierarchical B-spline meshes by statical condensation was proposed by Kagan et al. [20], and extended to NURBS meshes by Cottrell et al. [21]. This approach is limited to hierarchical meshes, which are a special case of non-conforming meshes. The method can be used for local refinement, but not for the coupling of arbitrary non-conforming patches. Thus, it is not considered to be a domain decomposition method.

The formulation proposed in this work represents an application of the mortar method as proposed by Bernardi et al. [10] in the frame of isogeometric analysis. The derivation and notation used within this contribution differs from [10], but the theoretical background is the same. The main idea was to derive a relation matrix which allows a connection of patches by statical condensation, akin to [20,21], but for the general case of non-conforming meshes. The relation matrix is attained with the help of a discretization of the matching conditions along the interface. The statical condensation with the derived relation matrix constrains the solutions of the subdomains to be equal along the interface. The difference to the mortar method proposed by Bernardi et al. [10] is that in our case the global system of equations is coupled with the help of a statical condensation instead of constraining the basis functions. However, the basic idea is the same. To the authors' knowledge, this work is the first application of the mortar method according to Bernardi et al. [10] in isogeometric analysis. The main features of the proposed formulation are:

(i) The basic aim is to enforce the equality of mutual deformations along the interface of adjacent patches. Basing on this equality a master-slave relation is derived. With the help of this relation the interface degrees of freedom of the slave patch can be expressed as a function of the interface degrees of freedom of the master patch. The slave interface degrees of freedom can be condensated out of the global system of equations. Thus, the domains are coupled in a natural way. The number of degrees of freedom is reduced.

(ii) The relation matrix is derived numerically. Unlike in [20,21] the method works for all kinds of non-conforming patch interfaces. The relation matrix has to be computed once as a preprocess with very little numerical effort. The method is applicable for nonlinear computations without additional effort for the equilibrium iteration.

(iii) The equality of mutual deformations is enforced in a weak manner. A variational equation is established from which the master-slave relation matrix can be attained. NURBS basis functions are defined over the whole patch and their mathematical properties guarantee their linear independence. This implies that the matrix inversion required for the computation of the relation matrix is well-defined.

(iv) The variational formulation is not altered. The connection is established by a statical condensation of the slave interface degrees of freedom. Thus, the method can be used for all kinds of formulations without further investigations about possible interactions. It is shown that the condition of the stiffness matrix does not deteriorate, as it is the case for some of the domain decomposition methods mentioned above. Nonlinear and dynamic problems can be handled without further measures.

The weak substitution method uses a very simple concept and the implementation is straightforward. Its scope is the connection of patches in nonlinear structural and solid mechanics. All other domain decomposition methods mentioned are more complex and have wider fields of application, such as contact mechanics.

It is to be remarked, that the presented method aims at enforcing $C^{0}$-continuity between patches, which suffices for Reissner-Mindlin shell elements, see [22, 23, 24]. In the proposed form, the method is not able to enforce $C^{1}$ continuity between patches, which is required for Kirchhoff-Love shell formulations [25]. The enforcement of the equality of mutual deformations with a collocation scheme instead of the weak form was tested by the authors and reported in [26]. No fully satisfying results were attained.

\section{Definition of the model problem: 2D plane stress analysis}

The proposed domain decomposition methods are derived for a 2D plane stress model problem.

\subsection{Domain decomposition}

For the computational implementation of our model problem we decompose the domain

$$
\Omega=\Omega^{(1)} \cup \Omega^{(2)} \cup \ldots \cup \Omega^{\left(n_{p}\right)}
$$


into $n_{p}$ subdomains. Without loss of generality we restrict the derivation to the case of two subdomains. The presented methods are restricted to the non-overlapping case, where

$$
\Gamma_{c}=\Omega^{(1)} \cap \Omega^{(2)}
$$

is the interface curve. The domain is decomposed into subdomains in order to allow completely independent discretizations for adjacent patches, see Fig. 1. We define three cases for the connection of patches:

- conforming meshes. If the NURBS degree and the knots along the interface of two adjacent patches are equivalent, then the meshes are conforming. The control points along the interface coincide. The two patches can be coupled by linking the degrees of freedom of every pair of coinciding control points.

- hierarchical meshes. If one knot vector along the interface is completely contained in its adjacent counterpart and the NURBS degree is equal, then the meshes are hierarchical. This means, that the finer mesh can be attained from the coarser by knot insertion. An exact relation between the control points on both sides exists for this case and is given in [20,21]. The control points on the refined side can be expressed as a function of the control points on the coarse side. Thus, the control points of the refined side can be removed from the system and a natural coupling of the patches is attained.

- non-conforming meshes. If the NURBS degree and the knot vector are arbitrary on both sides, then the meshes are non-conforming. No natural and exact connection is possible.

For the general case of non-conforming meshes two methods to couple adjacent patches are derived in the following.

\subsection{Strong form of the problem}

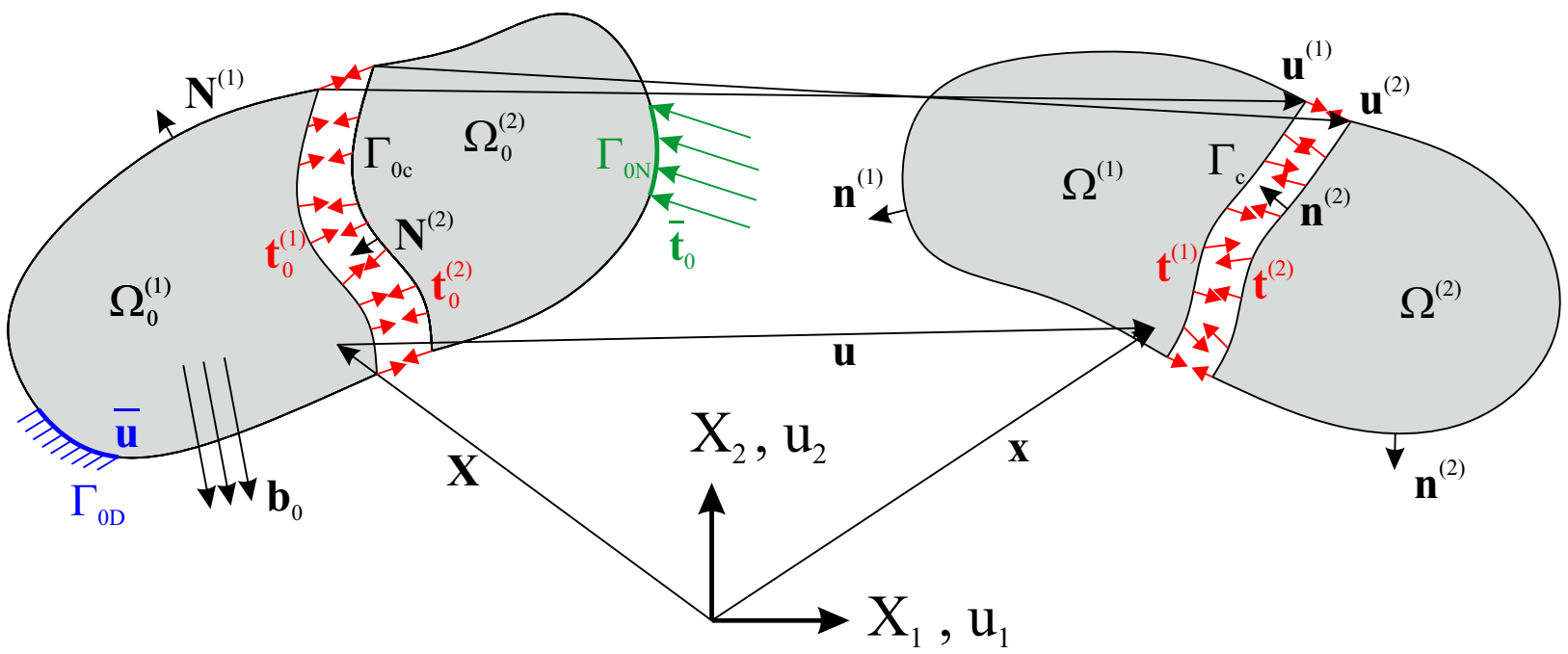

Figure 1: Reference and current configuration of the decomposed boundary value problem

An elastic body $\bar{\Omega}$ in a 2D plane stress setting is used as model problem. The domain $\Omega$ has the piecewise $C^{1}$ continuous boundary $\Gamma=\partial \Omega$ in the current configuration. Respective values in the reference configuration are labeled with a subscript 0 . Thus, the reference and the current unit normal vector $\mathbf{N}$ respective $\mathbf{n}$ with respect to the boundary is defined uniquely everywhere, except at kinks with $C^{0}$-continuity. Dirichlet boundary conditions $\mathbf{u}=\overline{\mathbf{u}}=\mathbf{0}$ are applied on $\Gamma_{0 D}$. The derivation is restricted to the homogenous case without loss of generality. Conservative Neumann boundary conditions $\mathbf{t}_{0}=\overline{\mathbf{t}}_{0}$ are applied on $\Gamma_{0 N}$ and do not overlap with the Dirichlet conditions, i.e. $\Gamma_{0 D} \cap \Gamma_{0 N}=\emptyset$. The reference configuration $\Omega_{0}$ is described by particle points $\mathbf{X}=(x, y) \in \Omega_{0}$. The current configuration of this point is determined with

$$
\mathbf{x}=\mathbf{X}+\mathbf{u}
$$


In order to allow nonlinear computations with large deformations, the strains are described with the Green-Lagrange strain tensor

$$
\mathbf{E}=\frac{1}{2}\left(\mathbf{F}^{T} \mathbf{F}-\mathbf{1}\right) \quad \text { in } \Omega_{0}
$$

with the deformation gradient $\mathbf{F}=\operatorname{Grad} \mathbf{x}=\frac{\partial \mathbf{x}}{\partial \mathbf{x}}$. Here $\mathbf{1}$ denotes the unity matrix. This results in

$$
\begin{aligned}
\mathbf{E}= & E_{i j} \mathbf{e}^{i} \otimes \mathbf{e}^{j} \\
& E_{i j}=\frac{1}{2}\left(\mathbf{x}_{, i} \cdot \mathbf{x}_{, j}-\mathbf{X}_{, i} \cdot \mathbf{X}_{, j}\right),
\end{aligned}
$$

where $\mathbf{e}^{i}$ are global Cartesian basis vectors and subscript commas denote derivation with respect to the global direction $X_{i}$. Latin indices range from one to two. Accordingly, the stresses are measured with the $2^{\text {nd }}$ Piola-Kirchhoff stress tensor $\mathbf{S}$. The stresses are computed using a linear St. Venant-Kirchhoff material model

$$
\mathbf{S}=\mathbb{C}: \mathbf{E} \text { in } \Omega_{0},
$$

where $\mathbb{C}$ is the elasticity tensor. Due to the confinement to a $2 \mathrm{D}$ plane stress problem the stresses are constant over the thickness $h$ of the body. Traction forces $\mathbf{t}$ at the boundaries are computed with the relation

$$
\mathbf{t}=h(\boldsymbol{\sigma} \cdot \mathbf{n}) \quad \text { on } \Gamma
$$

for the current configuration and

$$
\mathbf{t}_{0}=h(\mathbf{P} \cdot \mathbf{N}) \quad \text { on } \Gamma_{0}
$$

for the reference configuration. Here the Cauchy stress tensor $\boldsymbol{\sigma}=\mathbf{F S}^{T} \mathbf{F}^{T} / \operatorname{det} \mathbf{F}$ and the $1^{\text {st }}$ Piola-Kirchhoff stress tensor $\mathbf{P}=\mathbf{F S}$ is used, respectively. With the Neumann and Dirichlet conditions the strong form of the boundary value problem for the complete domain reads

$$
\begin{aligned}
\operatorname{Div} \mathbf{P}+\mathbf{b}_{0} & =\mathbf{0} & & \text { in } \Omega_{0} \\
\mathbf{t}_{0} & =\overline{\mathbf{t}}_{0} & & \text { on } \Gamma_{0 N} \\
\mathbf{u} & =\overline{\mathbf{u}} & & \text { on } \Gamma_{0 D},
\end{aligned}
$$

where $\mathbf{b}_{0}$ are the body forces acting upon $\Omega_{0}$. The Neumann conditions (9b) are applied on the reference configuration $\Gamma_{0 N}$. The domain $\Omega$ is decomposed into two subdomains $\Omega^{(1)}$ and $\Omega^{(2)}$, for which (1) and (2) holds. Boundary conditions, displacements, stresses and tractions are decomposed akin. If present, the superscript $(k)$ denotes the domain on which the quantity is defined. The interface $\Gamma_{c}$ is the connection between the two subdomains. It is a piecewise $C^{1}$-continuous curve. The boundary value problem (9) is divided along $\Gamma_{c}$ into two independent boundary value problems. Thus, additional coupling conditions have to be introduced. The equality of mutual displacements along the interface

$$
\mathbf{u}^{(1)}=\mathbf{u}^{(2)} \quad \text { on } \Gamma_{0 c}
$$

has to be enforced. This constraint transforms the two independent stationary problems into one constrained stationary problem. The interface tractions have to fulfill

$$
\mathbf{t}_{0}^{(1)}+\mathbf{t}_{0}^{(2)}=\mathbf{0} \quad \text { on } \Gamma_{0 c} .
$$

If the boundary value problem (9) is fulfilled inside every domain and the displacement equality (10) holds, then (11) naturally holds. Thus, the imposition of (11) is not necessary, but may lead to a smoother distribution of stresses in the context of an isogeometric approximation with NURBS elements. In common $C^{0}$-continuous finite element formulations the equality of stresses is not enforced between elements. Considering the initial boundary value problem (9) and the interface conditions (10) and (11), the decomposed boundary value problem reads

$$
\begin{array}{rlrl}
\operatorname{Div} \mathbf{P}+\mathbf{b}_{0} & =\mathbf{0} & & \text { in } \Omega_{0}=\Omega_{0}^{(1)}+\Omega_{0}^{(2)} \\
\mathbf{t}_{0} & =\overline{\mathbf{t}}_{0} & & \text { on } \Gamma_{0 N}=\Gamma_{0 N}^{(1)}+\Gamma_{0 N}^{(2)} \\
\mathbf{u} & =\overline{\mathbf{u}} & & \text { on } \Gamma_{0 D}=\Gamma_{0 D}^{(1)}+\Gamma_{0 D}^{(2)} \\
\mathbf{u}^{(1)}-\mathbf{u}^{(2)}=\mathbf{0} & & \text { on } \Gamma_{0 c} \\
\mathbf{t}_{0}^{(1)}+\mathbf{t}_{0}^{(2)}=\mathbf{0} & & \text { on } \Gamma_{0 c} .
\end{array}
$$




\subsection{Weak form of the problem}

The weak form of the boundary value problem (12) is deduced without consideration of the interface conditions (12d) and (12e). The weak form corresponds to the variation of the potential. For the two patches $k$ with $k=1,2$ it reads

$$
\partial \Pi(\mathbf{u}, \delta \mathbf{u})=\sum_{k=1}^{2}\left(\int_{\Omega_{0}^{(k)}} \mathbf{S}(\mathbf{u}): \delta \mathbf{E}(\delta \mathbf{u}) \mathrm{d} \Omega-\int_{\Gamma_{0 N}^{(k)}} \overline{\mathbf{t}}_{0} \cdot \delta \mathbf{u} \mathrm{d} \Gamma-\int_{\Omega_{0}^{(k)}} \mathbf{b}_{0} \cdot \delta \mathbf{u} \mathrm{d} \Omega\right)=0
$$

and fulfills (12a) and (12b). The Dirichlet boundary conditions have to be built into the solution space. The solution space $\mathcal{S}$ is defined to be

$$
\mathcal{S}=\left\{\mathbf{u} \in\left(H^{1}\left(\Omega^{(1)} \cup \Omega^{(2)}\right)\right)^{2} \mid \mathbf{u}=\mathbf{0} \text { on } \Gamma_{0 D}\right\}
$$

and thus (12c) holds by definition. Finally, a value $\mathbf{u} \in \mathcal{S}$ that fulfills

$$
\partial \Pi(\mathbf{u}, \delta \mathbf{u})=0 \quad \forall \delta \mathbf{u} \in \mathcal{S}
$$

has to be found. The variation of the Green-Lagrange strain tensor is analogously to (5) defined as

$$
\begin{aligned}
\delta \mathbf{E}= & \delta E_{i j} \mathbf{e}^{i} \otimes \mathbf{e}^{j} \\
& \delta E_{i j}=\frac{1}{2}\left(\delta \mathbf{x}_{, i} \cdot \mathbf{x}_{, j}+\mathbf{x}_{, i} \cdot \delta \mathbf{x}_{, j}\right) .
\end{aligned}
$$

Nonlinear computations with the Newton-Raphson scheme require the linearization of the weak form (13). The equation

$$
\mathrm{L}[\delta \Pi(\mathbf{u}, \delta \mathbf{u})]=\delta \Pi(\mathbf{u}, \delta \mathbf{u})+\mathrm{D} \delta \Pi(\mathbf{u}, \delta \mathbf{u}) \cdot \Delta \mathbf{u}=0
$$

with

$$
\mathrm{D} \delta \Pi(\mathbf{u}, \delta \mathbf{u}) \cdot \Delta \mathbf{u}=\sum_{k=1}^{2}\left(\int_{\Omega_{0}^{(k)}}(\mathbb{C}: \Delta \mathbf{E}): \delta \mathbf{E}+\mathbf{S}: \Delta \delta \mathbf{E} \mathrm{d} \Omega\right)
$$

has to be solved. Incremental values $\Delta(\ldots)$ are attained by replacing $\delta$ with $\Delta$. The second variation of the GreenLagrange strain tensor reads

$$
\begin{aligned}
\Delta \delta \mathbf{E}= & \Delta \delta E_{i j} \mathbf{e}^{i} \otimes \mathbf{e}^{j} \\
& \Delta \delta E_{i j}=\frac{1}{2}\left(\delta \mathbf{x}_{, i} \cdot \Delta \mathbf{x}_{, j}+\Delta \mathbf{x}_{, i} \cdot \delta \mathbf{x}_{, j}\right) .
\end{aligned}
$$

\subsection{Nomenclature for multi-patch NURBS surfaces}

Bivariate NURBS surface patches are used to describe the geometry. The number of control points of the patch $k$ is given by $n_{n p}^{(k)}=n_{1}^{(k)} \times n_{2}^{(k)}$, where $n_{\alpha}^{(k)}$ is the number of control points in the parametric direction $\alpha$. Analogously, the degree $p_{\alpha}^{(k)}$ in the parametric direction $\alpha$ is defined. The terms degree and order are equivalently used to refer to $p_{\alpha}^{(k)}$. A control point $\mathbf{B}_{i j}^{(k)}$ is defined by its two physical coordinates $x_{i j k}$ and $y_{i j k}$, which are assembled into the vector $\mathbf{X}^{(i, j, k)}$, and its weight factor $w_{i j k}$. Besides the set of $n_{n p}^{(k)}$ control points

$$
\mathbf{B}_{i j}^{(k)}=\left[x_{i j k}, y_{i j k}, w_{i j k}\right]^{T}=\left[\begin{array}{c}
\mathbf{X}^{(i, j, k)} \\
w_{i j k}
\end{array}\right] \quad i=1, \ldots, n_{1}^{(k)} \quad j=1, \ldots, n_{2}^{(k)},
$$

the two open knot vectors

$$
\boldsymbol{\Xi}_{\alpha}^{(k)}=\left\{\xi_{1}^{\alpha}, \xi_{2}^{\alpha}, \cdots, \xi_{n_{\alpha}^{(k)}+p_{\alpha}^{(k)}+1}^{\alpha}\right\} \quad \alpha=1,2,
$$

are required to define the patch $k$. The bijective function

$$
f(i, j, k)=I
$$


specifies the node index $I$ of the control point $\mathbf{B}_{i j}^{(k)}$ for the isogeometric implementation. The vector

$$
\mathbf{I}^{(k)}=\left\{f(i, j, k) \in \mathbb{N} \mid i=1, \ldots, n_{1}^{(k)} ; j=1, \ldots, n_{2}^{(k)}\right\}
$$

contains the node indices of all control points of patch $k$. With the help of (22) a physical point $\mathbf{X}$ on the domain $\Omega_{0}$ is interpolated with

$$
\mathbf{X}^{h}\left(\xi^{1}, \xi^{2}, k\right)=\sum_{I=1}^{n_{e n}^{(k)}} N_{I}\left(\xi^{1}, \xi^{2}, k\right) \mathbf{X}_{I}
$$

using the NURBS basis functions $N_{I}$. The number of basis functions having influence on an arbitrary element is specified by $n_{e n}^{(k)}=\left(p_{1}^{(k)}+1\right)\left(p_{2}^{(k)}+1\right)$. The NURBS basis functions $N_{I}$ are determined by

$$
N_{I}\left(\xi^{1}, \xi^{2}, k\right)=\frac{N_{i}^{p_{1}^{(k)}}\left(\xi^{1}\right) N_{j}^{p_{2}^{(k)}}\left(\xi^{2}\right) w_{i j k}}{\sum_{i=1}^{n_{1}^{(k)}} \sum_{j=1}^{n_{j}^{(k)}} N_{i}^{p_{1}^{(k)}}\left(\xi^{1}\right) N_{j}^{p_{2}^{(k)}}\left(\xi^{2}\right) w_{i j k}}
$$

using the B-spline basis functions $N_{i}^{p_{1}^{(k)}}$ and $N_{j}^{p_{2}^{(k)}}$. For more details about the computation of the basis functions and its derivatives we refer to $[2,3]$.

\subsection{Discrete form of the uncoupled problem}

The uncoupled model problem expressed by the linearization of the weak form (17) is discretized with NURBS basis functions. The reference and the current configuration are interpolated with

$$
\mathbf{X}^{h}=\sum_{I=1}^{n_{e n}^{(k)}} N_{I} \mathbf{X}_{I} \quad \mathbf{x}^{h}=\sum_{I=1}^{n_{e n}^{(k)}} N_{I} \mathbf{x}_{I}
$$

using the basis functions $N_{I}$ defined in (25) and discrete values $\mathbf{X}_{I}$ and $\mathbf{x}_{I}$. The superscript $h$ identifies interpolated values. The derivatives of (26) read

$$
\mathbf{X}_{, \alpha}^{h}=\sum_{I=1}^{n_{c n}^{(k)}} N_{I, \alpha} \mathbf{X}_{I} \quad \mathbf{x}_{, \alpha}^{h}=\sum_{I=1}^{n_{e n}^{(k)}} N_{I, \alpha} \mathbf{x}_{I},
$$

where $N_{I, \alpha}$ are the derivatives of the basis functions with respect to global Cartesian coordinates. Greek indices range from 1 to 2 if not specified differently. The variations of $\mathbf{x}^{h}$ and $\mathbf{x}_{, \alpha}^{h}$ are interpolated akin with

$$
\delta \mathbf{x}^{h}=\sum_{I=1}^{n_{e n}^{(k)}} N_{I} \delta \mathbf{u}_{I} \quad \delta \mathbf{x}_{, \alpha}^{h}=\sum_{I=1}^{n_{e n}^{(k)}} N_{I, \alpha} \delta \mathbf{u}_{I} .
$$

The interpolation of the variation of the strains in Voigt notation thus reads

$$
\delta \mathbf{E}^{h}=\left[\begin{array}{c}
\delta E_{11}^{h} \\
\delta E_{22}^{h} \\
2 \delta E_{12}^{h}
\end{array}\right]=\sum_{I=1}^{n_{e n}^{(k)}} \mathbf{B}_{I} \delta \mathbf{u}_{I}=\sum_{I=1}^{n_{e n}^{(k)}}\left[\begin{array}{c}
N_{I, 1} \mathbf{x}_{11}^{h T} \\
N_{I, 2} \mathbf{x}_{, 2} \\
N_{I, 1} \mathbf{x}_{, 2}^{h T}+N_{I, 2} \mathbf{x}_{, 1}^{h T}
\end{array}\right] \delta \mathbf{u}_{I},
$$

where the matrices $\mathbf{B}_{I}$ establish a relation between nodal deformations and strains. Incremental and virtual nodal deformations of every patch $k$ are assembled in the vectors

$$
\Delta \widetilde{\mathbf{u}}^{(k)}=\left[\Delta \mathbf{u}_{1}^{T}, \Delta \mathbf{u}_{2}^{T}, \ldots, \Delta \mathbf{u}_{n_{n p}^{(k)}}^{T}\right]^{T}
$$


respectively $\delta \widetilde{\mathbf{u}}^{(k)}$ following the same scheme. The patch wise approximation of (17)

$$
\mathrm{L}[\delta \Pi(\mathbf{u}, \delta \mathbf{u})]=\bigcup_{k=1}^{n_{p}} \delta \widetilde{\mathbf{u}}^{(k)^{T}}\left(\mathbf{K}^{(k)} \Delta \widetilde{\mathbf{u}}^{(k)}-\mathbf{f}^{(k)}\right)=0
$$

with

$$
\mathbf{K}^{(k)}=\bigcup_{e=1}^{n_{e l}^{(k)}} \sum_{I=1}^{n_{n=1}^{(k)}} \sum_{K=1}^{n_{e n}^{(k)}} \mathbf{K}_{I K}^{e} \quad \mathbf{f}^{(k)}=\bigcup_{e=1}^{n_{e l}^{(k)}} \sum_{I=1}^{n_{e n}^{(k)}} \mathbf{f}_{I}^{e}
$$

results in an uncoupled system of equations

$$
\left[\begin{array}{cc}
\mathbf{K}^{(1)} & \mathbf{0} \\
\mathbf{0} & \mathbf{K}^{(2)}
\end{array}\right]\left[\begin{array}{c}
\Delta \widetilde{\mathbf{u}}^{(1)} \\
\Delta \widetilde{\mathbf{u}}^{(2)}
\end{array}\right]=\left[\begin{array}{l}
\mathbf{f}^{(1)} \\
\mathbf{f}^{(2)}
\end{array}\right]
$$

The solution space for $\Delta \widetilde{\mathbf{u}}^{(k)}$ in (33) has to be a subspace of $\mathcal{S}$ defined in (14). Using Voigt notation for the strains $\mathbf{E}$, the stresses $\mathbf{S}$ and the material matrix $\mathbf{C}$, the element stiffness matrices are computed by

$$
\mathbf{K}_{I K}^{e}=\int_{\Omega_{0}^{e}}\left(\mathbf{B}_{I}^{T} \mathbf{C B}_{K}+\mathbf{G}_{I K}\right) \mathrm{d} \Omega,
$$

where $\mathbf{G}_{I K}$ denotes the initial stress stiffness matrix

$$
\mathbf{G}_{I K}=\mathbf{1}\left(S^{11} N_{I, 1} N_{K, 1}+S^{22} N_{I, 2} N_{K, 2}+S^{12}\left(N_{I, 1} N_{K, 2}+N_{I, 2} N_{K, 1}\right)\right) .
$$

Here 1 denotes the $2 \times 2$ unity matrix. The element load vector reads

$$
\mathbf{f}_{I}^{e}=\mathbf{f}_{I}^{e, e x t}-\int_{\Omega_{0}^{e}} \mathbf{B}_{I}^{T} \mathbf{S} \mathrm{d} \Omega
$$

where the external load vector $\mathbf{f}_{I}^{e, e x t}$ is defined by the second and third term in (13).

\section{Techniques for domain coupling/decomposition}

The weak form derived in Sec. 2.3 describes the uncoupled boundary value problem constituted by (12a)-(12c). Two methods to enforce the additional equations (12d) and (12e) are presented in the following. The Lagrange multiplier method is a standard method for domain coupling and thus only briefly summarized. The implementation serves as a benchmark method. The weak substitution method proposed in Sec. 3.2 is a new concept to connect non-conforming patches.

\subsection{Lagrange multiplier method}

The Lagrange multiplier method is a widespread computational strategy to solve equations with constraints. The constraint is defined by a constraint function $\mathbf{f}_{L M}$. The constraint function is multiplied with an unknown variable field $\lambda$ and integrated over the interface $\Gamma_{0 c}$. This product is added to the potential $\Pi$. The variation of the enhanced potential has to be zero. By adding the additional terms to the potential the stationary problem is transformed into a saddle point problem.

\subsubsection{Variational formulation for domain coupling}

The equality of mutual deformations (12d) along the interface $\Gamma_{0 c}$ is used as constraint function $\mathbf{f}_{L M}=\mathbf{u}^{(1)}-\mathbf{u}^{(2)}$ and thus

$$
\Pi_{L M}^{+}=\eta \int_{\Gamma_{0 c}} \lambda \cdot\left(\mathbf{u}^{(1)}-\mathbf{u}^{(2)}\right) \mathrm{d} \Gamma
$$

is the additional term for the potential. The multiplicative constant $\eta$ scales the Lagrange multiplier terms and thus allows to control the condition of the global stiffness matrix. The variation of (37) results in two additional terms 
introducing the additional variables $\lambda \in \mathcal{L}$ and their variations $\delta \lambda \in \mathcal{L}$ into (13). The space $\mathcal{L}$ is defined by $\mathcal{L}=$ $\left(L^{2}\left(\Gamma_{c}\right)\right)^{2}$. The restriction $\mathbf{u} \in \mathcal{T}$, where

$$
\mathcal{T}=\left\{\mathbf{u} \in \mathcal{S} \mid\left(\mathbf{u}^{(1)}-\left.\mathbf{u}^{(2)}\right|_{\Gamma_{c}}\right) \in \mathcal{L}\right\}
$$

is a subset of $\mathcal{S}$, precludes infinite values for the integral in (37). The complete weak form of the coupled problem using the Lagrange multiplier method reads

$$
\begin{aligned}
\partial \Pi_{L M}(\mathbf{u}, \delta \mathbf{u}, \lambda, \delta \boldsymbol{\lambda}) & =\sum_{k=1}^{2}\left(\int_{\Omega_{0}^{(k)}} \mathbf{S}: \delta \mathbf{E} \mathrm{d} \Omega-\int_{\Gamma_{0 N}^{(k)}} \overline{\mathbf{t}}_{0} \cdot \delta \mathbf{u} \mathrm{d} \Gamma-\int_{\Omega_{0}^{(k)}} \mathbf{b}_{0} \cdot \delta \mathbf{u} \mathrm{d} \Omega\right) \\
& +\eta \int_{\Gamma_{0 c}} \lambda \cdot\left(\delta \mathbf{u}^{(1)}-\delta \mathbf{u}^{(2)}\right) \mathrm{d} \Gamma+\eta \int_{\Gamma_{0 c}} \delta \lambda \cdot\left(\mathbf{u}^{(1)}-\mathbf{u}^{(2)}\right) \mathrm{d} \Gamma .
\end{aligned}
$$

Finally, values $\mathbf{u} \in \mathcal{T}$ and $\lambda \in \mathcal{L}$ that fulfill

$$
\partial \Pi_{L M}(\mathbf{u}, \delta \mathbf{u}, \lambda, \delta \lambda)=0 \quad \forall \delta \mathbf{u} \in \mathcal{T}, \delta \lambda \in \mathcal{L}
$$

have to be found. The variational formulation (40) constitutes a saddle point problem with the solution space $\mathcal{V}=$ $\mathcal{T} \times \mathcal{L}$. Thus, the inf-sup conditions [8] have to be fulfilled. For more details see [17]. The linearization

$$
\begin{aligned}
\mathrm{L}[\delta \Pi(\mathbf{u}, \delta \mathbf{u}, \lambda, \delta \lambda)] & =\delta \Pi(\mathbf{u}, \delta \mathbf{u}, \lambda, \delta \lambda) \\
& +\mathrm{D} \delta \Pi(\mathbf{u}, \delta \mathbf{u}, \lambda, \delta \lambda) \cdot \Delta \mathbf{u}+\mathrm{D} \delta \Pi(\mathbf{u}, \delta \mathbf{u}, \lambda, \delta \lambda) \cdot \Delta \lambda=0
\end{aligned}
$$

of the weak form (39) is required for nonlinear computations. The terms in (41) are given by

$$
\begin{aligned}
\mathrm{D} \delta \Pi(\mathbf{u}, \delta \mathbf{u}, \lambda, \delta \lambda) \cdot \Delta \mathbf{u} & =\sum_{k=1}^{2}\left(\int_{\Omega_{0}^{(k)}}(\mathbb{C}: \Delta \mathbf{E}): \delta \mathbf{E}+\mathbf{S}: \Delta \delta \mathbf{E} \mathrm{d} \Omega\right) \\
& +\eta \int_{\Gamma_{0 c}} \delta \boldsymbol{\lambda} \cdot\left(\Delta \mathbf{u}^{(1)}-\Delta \mathbf{u}^{(2)}\right) \mathrm{d} \Gamma
\end{aligned}
$$

and

$$
\mathrm{D} \delta \Pi(\mathbf{u}, \delta \mathbf{u}, \lambda, \delta \lambda) \cdot \Delta \lambda=\eta \int_{\Gamma_{0 c}} \Delta \lambda \cdot\left(\delta \mathbf{u}^{(1)}-\delta \mathbf{u}^{(2)}\right) \mathrm{d} \Gamma .
$$

\subsubsection{Isogeometric discretization}

A Lagrange multiplier field has to be defined for the discretization. In every patch only one row of control points has influence along the interface $\Gamma_{0 c}$. The quantity of these control points is

$$
n_{\Gamma}^{(k)}=\# \widetilde{\mathbf{X}}_{c}^{(k)} \quad \text { with } \quad \widetilde{\mathbf{X}}_{c}^{(k)}=\left\{\mathbf{B}_{i j}^{(k)}\left|N_{I} \neq 0\right|_{\Gamma_{0 c}^{(k)}} ; I \in \mathbf{I}^{(k)}\right\}
$$

for every patch $k$. The number of Lagrange multipliers is determined by $n_{\Gamma}=\min \left(n_{\Gamma}^{(1)}, n_{\Gamma}^{(2)}\right)$. The degree and the knot vector in interface direction of the patch with $n_{\Gamma}^{(k)}=n_{\Gamma}$ are used for the definition of the Lagrange multiplier field. Its interpolation is akin to (26)

$$
\lambda^{h}=\sum_{I=1}^{n_{\Gamma}} N_{I}^{\lambda} \lambda_{I}
$$

using $n_{\Gamma}$ nodal Lagrange multipliers $\lambda_{I}$ and the associated NURBS basis functions $N_{I}^{\lambda}$. The incremental Lagrange multipliers are assembled in the vector

$$
\Delta \widetilde{\boldsymbol{\lambda}}^{(k)}=\left[\Delta \boldsymbol{\lambda}_{1}^{T}, \Delta \boldsymbol{\lambda}_{2}^{T}, \ldots, \Delta \boldsymbol{\lambda}_{n_{\Gamma}}^{T}\right]^{T} .
$$


They are the additional degrees of freedom in the system. The virtual Lagrange multipliers $\delta \widetilde{\lambda}^{(k)}$ are gathered akin. With the additional matrices

$$
\mathbf{Q}^{(k)}=\bigcup_{\Gamma_{0 c}} \sum_{I=1}^{n_{\Gamma}} \sum_{K=1}^{n_{c o n}^{(k)}} \int_{\Gamma_{0 c}^{e}} \mathbf{1} \eta N_{I}^{\lambda} N_{K} \mathrm{~d} \Gamma
$$

and the additional entries for the residuum vector

$$
\mathbf{R}^{(k)}=\bigcup_{\Gamma_{0 c}} \sum_{I=1}^{n_{e n}^{(k)}} \int_{\Gamma_{0 c}^{e}} \eta N_{I} \lambda^{h} \mathrm{~d} \Gamma
$$

the discretization of (41) reads

$$
\left[\begin{array}{ccc}
\mathbf{K}^{(1)} & \mathbf{0} & \mathbf{Q}^{(1)} \\
\mathbf{0} & \mathbf{K}^{(2)} & -\mathbf{Q}^{(2)} \\
\mathbf{Q}^{(1)^{\mathrm{T}}} & -\mathbf{Q}^{(2)^{\mathrm{T}}} & \mathbf{0}
\end{array}\right]\left[\begin{array}{c}
\Delta \widetilde{\mathbf{u}}^{(1)} \\
\Delta \widetilde{\mathbf{u}}^{(2)} \\
\Delta \widetilde{\lambda}
\end{array}\right]=\left[\begin{array}{c}
\mathbf{f}^{(1)}-\mathbf{R}^{(1)} \\
\mathbf{f}^{(2)}+\mathbf{R}^{(2)} \\
\mathbf{0}
\end{array}\right] .
$$

\subsection{Weak substitution method}

A completely different approach for the coupling of patches is pursued for the derivation of the weak substitution method. The variational formulation is not altered. The connection is attained by establishing a relation between the degrees of freedom of adjacent edges. The deformations of one patch - the slave patch - are expressed as a function of the other patch - the master patch. For hierarchic knot vectors an analytical relation exists, see [20]. A relation for the general case is derived in the following. With the help of this relation the edge degrees of freedom of the slave patch can be removed from the global system of equations. Thus, the equality of mutual deformations (12d) holds and the patches are naturally connected. The system of equations of the uncoupled problem (33) is coupled by shared degrees of freedom without any further effort. The equality of mutual interface stresses (12e) is not enforced strongly. Akin to the standard procedure for the connection of conforming patches with shared degrees of freedom, $C^{0}$-continuity for the deformations at the interface is attained. Higher continuity is not required. The nature of the proposed method allows to use the method for all kinds of nonlinearities and more sophisticated element types, e.g. shells, without further measures.

\subsubsection{Mathematical derivation of the relation}

The domain $\Omega$ is decomposed into two subdomains $\Omega^{(1)}$ and $\Omega^{(2)}$ with the interface $\Gamma_{c}$. The tractions at both sides of the interface must be opposite with an equal absolute value to fulfill Newton's third law. Thus, every interface traction distribution

$$
\mathbf{t}_{c}(\mathbf{X})=\mathbf{P}^{(1)}(\mathbf{X}) \cdot \mathbf{N}^{(1)}(\mathbf{X})=-\mathbf{P}^{(2)}(\mathbf{X}) \cdot \mathbf{N}^{(2)}(\mathbf{X}) \quad \forall \mathbf{X} \in \Gamma_{0 c}
$$

along the interface $\Gamma_{0 c}$ describes the traction distribution along the interface for both patches. This is depicted in Fig. 2. The interface traction $\mathbf{t}_{c}$ is not an external load, only a virtual internal one. Thus, the work acted by $\mathbf{t}_{c}$ on the body $\bar{\Omega}$ must be identically zero for all $\mathbf{t}_{c} \in \mathcal{L}$, where $\mathcal{L}$ will be defined in (55). This requirement is expressed by

$$
\int_{\Gamma_{0 c}^{(1)}} \mathbf{t}_{c} \cdot \mathbf{u}^{(1)} \mathrm{d} \Gamma-\int_{\Gamma_{0 c}^{(2)}} \mathbf{t}_{c} \cdot \mathbf{u}^{(2)} \mathrm{d} \Gamma=0 \quad \forall \mathbf{t}_{c} \in \mathcal{L}
$$

with $\mathbf{u} \in \mathcal{T}$. Due to its virtual character, $\mathbf{t}_{c}$ can be regarded as a test function. Thus, (51) constitutes the weak form of the interface condition (12d). The vectors in (51) are interpolated with

$$
\mathbf{u}^{(k)}=\sum_{I=1}^{n_{\Gamma}^{(k)}} N_{I}^{(k)} \mathbf{u}_{I}^{(k)} \quad \mathbf{t}_{c}=\sum_{I=1}^{n_{\Gamma}} N_{I}^{(t)} \mathbf{t}_{I}
$$

using NURBS basis functions. The number of control points having influence along the interface $n_{\Gamma}^{(k)}$ is defined by (44), whereas $n_{\Gamma}$ will be defined later on. At this point a classification of the patches in master and slave is necessary. The superscript $(\mathrm{ma})$ denotes the master patch, while $(\mathrm{sl})$ denotes the slave patch. A general criterion for 

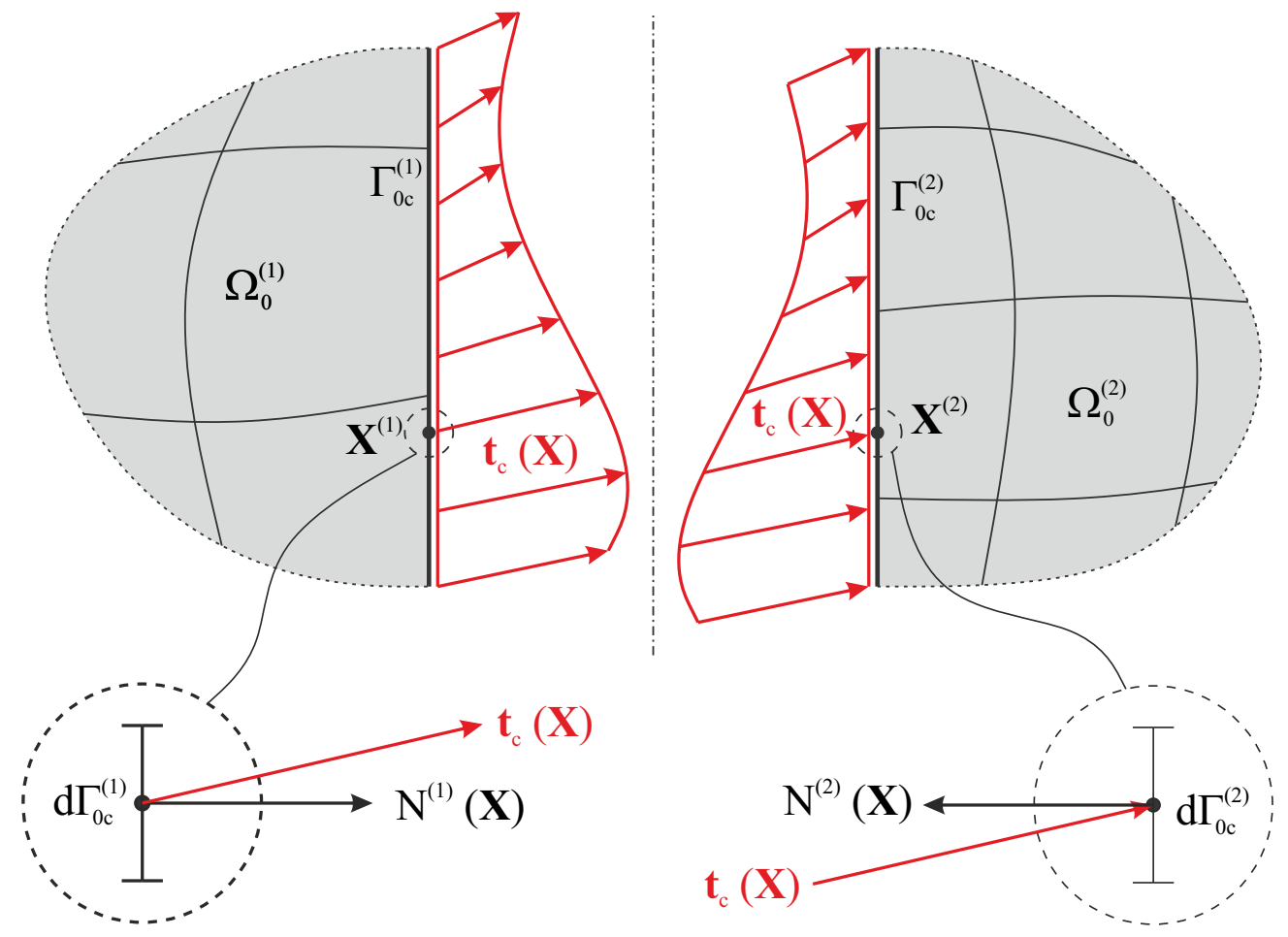

Figure 2: Interface tractions on a infinitesimal small line element.

the classification is not provided at this point. The two possible choices are examined in the numerical examples in Sec. 4. Computations with the weak substitution method using the criterion

$$
n_{\Gamma}^{(s l)}=\max \left(n_{\Gamma}^{(1)}, n_{\Gamma}^{(2)}\right) \quad n_{\Gamma}^{(m a)}=\min \left(n_{\Gamma}^{(1)}, n_{\Gamma}^{(2)}\right)
$$

are denoted by WSMa, the opposite choice

$$
n_{\Gamma}^{(s l)}=\min \left(n_{\Gamma}^{(1)}, n_{\Gamma}^{(2)}\right) \quad n_{\Gamma}^{(m a)}=\max \left(n_{\Gamma}^{(1)}, n_{\Gamma}^{(2)}\right)
$$

by WSMb. The number of nodal values for the virtual traction force $\mathbf{t}_{c}$ has to be equal to the number of interface control points in the slave patch, i.e. $n_{\Gamma}=n_{\Gamma}^{(s l)}$. The function space for the virtual traction force $\mathbf{t}_{c} \in \mathcal{L}$ is stated more precisely as

$$
\mathcal{L}=\left(L^{2}\left(\Gamma_{c}^{(s l)}\right)\right)^{2}
$$

The choice $\mathbf{t}_{c} \in \mathcal{L}$ simply uses the NURBS parametrization of the slave patch along the interface for the virtual traction force. Thus, each basis function $N_{I}^{(t)}$ of the virtual traction force is equal to the basis function $N_{I}^{(s l)}$ of the slave patch for $I=1, \ldots, n_{\Gamma}^{(s l)}$. The influence of the proposed connection method on the mechanical response analysis depends on the choice of the function space $\mathcal{L}$. The choice made within this work yields non-local support of the interface basis functions on the slave side. The implementation is simplified, as $N_{I}^{(t)}=N_{I}^{(s l)}$ for $I=1, \ldots, n_{\Gamma}^{(s l)}$ holds. In [12] the usage of dual basis functions is proposed in order to recover local support of basis functions. In [13] dual basis functions for Lagrange polynomials of arbitrary order are proposed. An example of dual basis functions for B-splines is given in [16]. The idea of using dual basis functions for the discretization of $\mathbf{t}_{c}$ in (51) seems to be promising, but this approach is not followed further in this work.

The interpolated form of (51) reads

$$
\sum_{I=1}^{n_{\Gamma}} \sum_{K=1}^{n_{\Gamma}^{(s l)}} \mathbf{t}_{I}^{T}\left(\int_{\Gamma_{0 c}^{(s l)}} N_{I}^{(t)} N_{K}^{(s l)} \mathrm{d} \Gamma\right) \mathbf{u}_{K}^{(s l)}=\sum_{I=1}^{n_{\Gamma}} \sum_{K=1}^{n_{\Gamma}^{(m a)}} \mathbf{t}_{I}^{T}\left(\int_{\Gamma_{0 c}^{(m a)}} N_{I}^{(t)} N_{K}^{(m a)} \mathrm{d} \Gamma\right) \mathbf{u}_{K}^{(m a)} .
$$


Rewritten to matrix form using

$$
\widetilde{\mathbf{t}}=\left[\mathbf{t}_{1}^{T}, \mathbf{t}_{2}^{T}, \ldots, \mathbf{t}_{n_{\Gamma}}^{T}\right]^{T} \quad \widetilde{\mathbf{u}}_{c}^{(k)}=\left[\mathbf{u}_{1}^{(k)^{T}}, \mathbf{u}_{2}^{(k)^{T}}, \ldots, \mathbf{u}_{n_{\Gamma}^{(k)}}^{(k)^{T}}\right]^{T}
$$

and the mortar matrices

$$
\mathbf{D}^{(k)}=\left[D_{I K}^{(k)} \mathbf{1}\right]_{I=1, \ldots, n_{\Gamma} ; K=1, \ldots, n_{\Gamma}^{(k)}} \quad D_{I K}^{(k)}=\int_{\Gamma_{0 c}^{(k)}} N_{I}^{(t)} N_{K}^{(k)} \mathrm{d} \Gamma,
$$

the interpolated form (56) reads

$$
\widetilde{\mathbf{t}} \mathbf{D}^{(s l)} \widetilde{\mathbf{u}}_{c}^{(s l)}=\widetilde{\mathbf{t}} \mathbf{D}^{(m a)} \widetilde{\mathbf{u}}_{c}^{(m a)} .
$$

The index $c$ for the vector of the nodal deformations $\widetilde{\mathbf{u}}_{c}^{(k)}$ denotes that it contains the deformations of all interface control points. The traction vector $\widetilde{\mathbf{t}}$ acts as a test function. It can be dropped and thus the final equation

$$
\mathbf{D}^{(s l)} \widetilde{\mathbf{u}}_{c}^{(s l)}=\mathbf{D}^{(m a)} \widetilde{\mathbf{u}}_{c}^{(m a)}
$$

between the deformations in the master and in the slave patch is established. The mortar matrix $\mathbf{D}^{(s l)}$ is always square as $n_{\Gamma}=n_{\Gamma}^{(s l)}$ holds. The NURBS basis functions are linear independent, see [3]. The $n_{\Gamma}$ columns of the matrix $\mathbf{D}^{(s l)}$ contain a product of the NURBS basis functions. Thus, the $n_{\Gamma}$ columns are linear independent which implies that

$$
\operatorname{rank} \mathbf{D}^{(s l)}=n_{\Gamma} \cdot n_{\text {dim }}
$$

holds, where $n_{\text {dim }}$ is the dimension of the problem under consideration. Following [27], a well-defined inverse of the matrix $\mathbf{D}^{(s l)}$ exists. Thus, the final relation between the edge degrees of freedom of the master and the slave patch reads

$$
\widetilde{\mathbf{u}}_{c}^{(s l)}=\left(\mathbf{D}^{(s l)}\right)^{-1} \mathbf{D}^{(m a)} \widetilde{\mathbf{u}}_{c}^{(m a)}=\mathbf{T} \widetilde{\mathbf{u}}_{c}^{(m a)} .
$$

It holds accordingly for the location vectors $\widetilde{\mathbf{X}}^{(k)}$, the variations $\delta \widetilde{\mathbf{u}}^{(k)}$ and the increments $\Delta \widetilde{\mathbf{u}}^{(k)}$.

\subsubsection{Computation of the mortar matrices}

The computation of the mortar matrices (58) requires several projections. The integrals are evaluated in the parametric space of the master patch for $\mathbf{D}^{(m a)}$ and in the parametric space of the slave patch for $\mathbf{D}^{(s l)}$. In the case of the matrix $\mathbf{D}^{(m a)}$, the integral

$$
D_{I K}^{(m a)}=\int_{\Gamma_{0 c}^{(m a)}} N_{I}^{(t)} N_{K}^{(m a)} \mathrm{d} \Gamma
$$

contains the basis functions $N_{K}^{(m a)}$ defined on $\Gamma_{0 c}^{(m a)}$, and the basis functions $N_{I}^{(t)}$ defined on $\Gamma_{0 c}^{(s l)}$. Integration is performed in the parametric space of the master patch, which is spanned up by the two knot vectors $\boldsymbol{\Xi}_{1}^{(m a)} \otimes \boldsymbol{\Xi}_{2}^{(m a)}$. Every interface integration point

$$
\left(\xi_{m a}^{1}, \xi_{m a}^{2}\right)=\left\{\left(\xi^{1}, \xi^{2}\right) \in \mathbf{\Xi}_{1}^{(m a)} \otimes \boldsymbol{\Xi}_{2}^{(m a)} \mid \mathbf{X}^{h}\left(\xi^{1}, \xi^{2}, m a\right) \in \Gamma_{0 c}^{(m a)}\right\}
$$

in the parametric space of the master patch has to be projected onto the parametric space of the slave domain in order to evaluate the basis function $N_{I}^{(t)}$. The parametric spaces are not connected. Thus, the projection has to be performed in the physical space. For every interface point $\left(\xi_{m a}^{1}, \xi_{m a}^{2}\right)$ the physical location $\mathbf{X}^{(m a)}=\mathbf{X}^{h}\left(\xi_{m a}^{1}, \xi_{m a}^{2}, m a\right)$ has to be computed. The corresponding physical point $\mathbf{X}^{(s l)}$ on the slave patch has to be computed by a closest point projection, see [3]. The parameters $\left(\xi_{s l}^{1}, \xi_{s l}^{2}\right)$ of $\mathbf{X}^{(s l)}$ in the parametric space of the slave patch have to be determined with the help of the point inversion algorithm given in [3]. Thus, the interface point which corresponds to $\left(\xi_{m a}^{1}, \xi_{m a}^{2}\right)$ is defined by

$$
\left(\xi_{s l}^{1}, \xi_{s l}^{2}\right)=\left\{\left(\xi^{1}, \xi^{2}\right) \in \mathbf{\Xi}_{1}^{(s l)} \otimes \mathbf{\Xi}_{2}^{(s l)} \mid \mathbf{X}^{h}\left(\xi^{1}, \xi^{2}, s l\right)=\mathbf{X}^{(m a)}, \mathbf{X}^{h}\left(\xi^{1}, \xi^{2}, s l\right) \in \Gamma_{0 c}^{(s l)}\right\}
$$

in the slave parametric space, where the equality $\mathbf{X}^{h}\left(\xi^{1}, \xi^{2}, s l\right)=\mathbf{X}^{(m a)}$ is fulfilled up to numerical precision for watertight geometries. The equality can be replaced by a minimization of the distance $\mathbf{X}^{h}\left(\xi^{1}, \xi^{2}, s l\right)-\mathbf{X}^{(m a)}$ in case of 


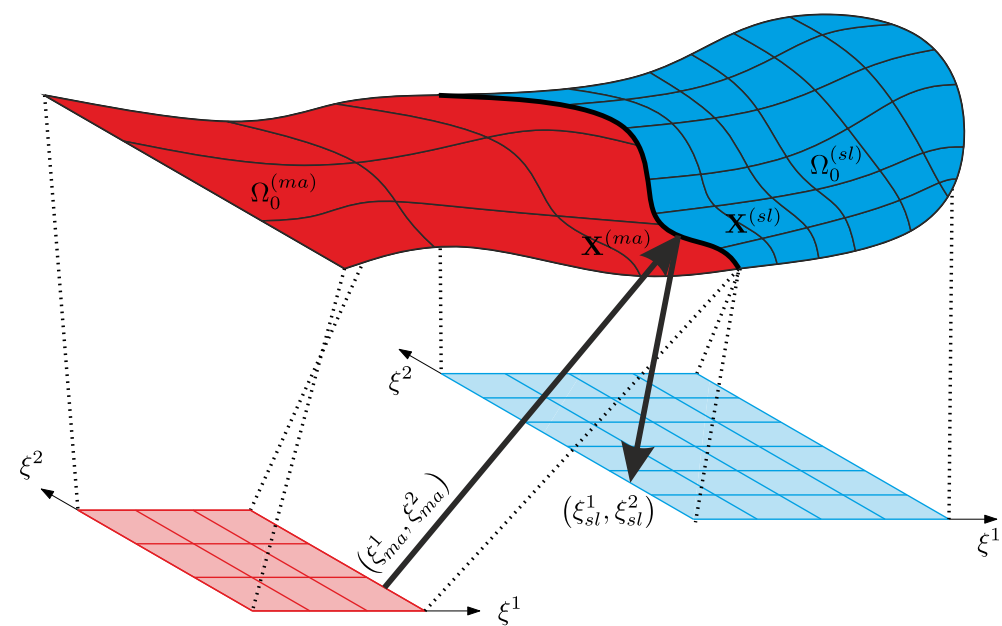

Figure 3: Required projections for the computation of the mortar integrals.

non-watertight geometries. The algorithmic treatment is the same in both cases; the closest point projection algorithm is used. The projections described above are sketched in Fig. 3. The computation of the matrix $\mathbf{D}^{(s l)}$ does not require any projection for the formulation at hand, as the function space of the virtual traction force is chosen to be equal to the slave patch.

B-spline basis function of degree $p$ can be integrated exactly within a knot span using Gauss integration with $p+1$ integration points. In order to ensure an accurate integration of $\mathbf{D}^{(s l)}$, Gauss integration has to be performed within every interface knot span of the slave patch. The computation of (63) requires that each knot value of the slave patch along the interface is projected onto the parametric space of the master patch. These projected knot values together with the knot values of the master patch define the integration spans $\Gamma_{\bar{e}}$ for (63). Thus, the integration of (63) can be accurately performed by

$$
D_{I K}^{(m a)}=\sum_{\bar{e}} \int_{\Gamma_{\bar{e}}} N_{I}^{(t)}\left(\xi_{s l}^{1}, \xi_{s l}^{2}\right) N_{K}^{(m a)}\left(\xi_{m a}^{1}, \xi_{m a}^{2}\right) \mathrm{d} \Gamma
$$

\subsubsection{Isogeometric discretization}

The equality of mutual deformations (12d) is enforced in an interpolated sense with the help of the relation matrix $\mathbf{T}$ defined in (62). The system (33) is coupled by replacing the interface degrees of freedom of the slave patch with the interface degrees of freedom of the master patch. Thus, the coupling of the patches is achieved by a substitution, where the substitution rule is attained by a weak form of the displacement continuity condition (12d). The substitution requires a split of the assembled deformation vectors $\widetilde{\mathbf{u}}^{(k)}$ into the interface deformation vector

$$
\widetilde{\mathbf{u}}_{c}^{(k)}=\left\{\mathbf{u}_{I}^{(k)}\left|N_{I} \neq 0\right|_{\Gamma_{c}^{(k)}} ; I \in \mathbf{I}^{(k)}\right\},
$$

which contains the deformations of all interface control points, and the domain deformation vector

$$
\widetilde{\mathbf{u}}_{d}^{(k)}=\left\{\mathbf{u}_{I}^{(k)}\left|N_{I}=0\right|_{\Gamma_{c}^{(k)}} ; I \in \mathbf{I}^{(k)}\right\} .
$$

The split

$$
\widetilde{\mathbf{u}}^{(k)}=\left[\begin{array}{c}
\widetilde{\mathbf{u}}_{d}^{(k)} \\
\widetilde{\mathbf{u}}_{c}^{(k)}
\end{array}\right]
$$

is equally performed for $\delta \widetilde{\mathbf{u}}^{(k)}$ and $\Delta \widetilde{\mathbf{u}}^{(k)}$ as well as for the load vector $\mathbf{f}^{(k)}$. Within the assembly of the stiffness matrix the split defined in (69) has to be considered. Thus, the patch wise defined stiffness matrix

$$
\mathbf{K}^{(k)}=\left[\begin{array}{cc}
\mathbf{K}_{d d}^{(k)} & \mathbf{K}_{d c}^{(k)} \\
\mathbf{K}_{c d}^{(k)} & \mathbf{K}_{c c}^{(k)}
\end{array}\right]
$$


is decomposed into four submatrices. The connected system of equations finally reads

$$
\left[\begin{array}{ccc}
\mathbf{K}_{d d}^{(m a)} & \mathbf{K}_{d c}^{(m a)} & \mathbf{0} \\
\mathbf{K}_{c d}^{(m a)} & \mathbf{K}_{c c}^{(m a)}+\mathbf{T}^{T} \mathbf{K}_{c c}^{(s l)} \mathbf{T} & \mathbf{T}^{T} \mathbf{K}_{c d}^{(s l)} \\
\mathbf{0} & \mathbf{K}_{d c}^{(s l)} \mathbf{T} & \mathbf{K}_{d d}^{(s l)}
\end{array}\right]\left[\begin{array}{c}
\Delta \widetilde{\mathbf{u}}_{d}^{(m a)} \\
\Delta \widetilde{\mathbf{u}}_{c}^{(m a)} \\
\Delta \widetilde{\mathbf{u}}_{d}^{(s l)}
\end{array}\right]=\left[\begin{array}{c}
\mathbf{f}_{d}^{(m a)} \\
\mathbf{f}_{c}^{(m a)} \\
\mathbf{f}_{d}^{(s l)}
\end{array}\right]
$$

The substitution eliminates all degrees of freedom assigned to the interface control points of the slave patch. Thus, the number of degrees of freedom is always reduced, which is not the case for the Lagrange multiplier method. The number of degrees of freedom depends on the classification of the patches to be master or slave.

\section{Numerical examples}

The accuracy and robustness of the proposed weak substitution method for the connection of NURBS surface patches is tested with the help of three numerical examples. The beam under pure tension yields a constant state of stress with known analytical solution. A discretization with one linear element already entails the exact solution. Thus, all encountered errors are due to the discretization with two patches with curved interface. This example is used to quantify the impact of the connection methods on the global convergence behavior and to compare the presented methods. The quarter of an annulus subject to tip shear force is a bending example with a known analytical solution. The geometry is curved and the load is applied as shear force. This example tests the performance of the connection methods for bending states. The elastic plate with hole is a standard benchmark for in-plane stress analysis with a complex but known analytical solution. Two different interfaces are considered, assessing both straight and curved patch intersection. In the linear case, the nature of the employed examples allows error norms to be used to compare the numerical results of the connection methods with analytical results. For this sake the $L_{2}$-norm, denoted by $\|\bullet\|_{0, \Omega}$, is used. The $L_{2}$-error norm $\left\|\mathbf{S}-\mathbf{S}^{h}\right\|_{0, \Omega}$ allows quantifying the impact of the connection methods on the stress distribution throughout the whole domain. Analytical solutions are not available for nonlinear computations. Thus, results are compared to precise computations with conforming meshes. Computations with the Lagrange multiplier method, which is presented in Sec. 3.1, are denoted by LM in the legend. Computations with the weak substitution method are labeled with WSMa or WSMb, depending on which patch is chosen to be the master patch, see Sec. 3.2.3. Computations of conforming meshes, which are connected by shared degrees of freedom, are labeled by DC. All integrals are evaluated numerically using Gauss integration with

$$
n_{g p}=\left(\max \left(p_{\alpha}^{(k)}\right)+1\right)^{n_{\text {dim }}}, \quad \alpha=1,2, \quad k=1,2
$$

integration points, where $n_{\text {dim }}$ is the dimension of the concerned integral.

\subsection{Beam with curved interface under pure tension}

This example yields a constant state of stress. An analytical solution can be found easily, as well as computations with one linear element entail the correct solution of the problem. The geometry is modeled with two NURBS patches in order to study the influence of the presented connection methods. The interface is described by a NURBS curve of which the details are given in Tab. 1. This discretization does not yield the exact solution for the coarsest mesh due to the rational character of the NURBS basis functions. The employed Gauss integration scheme is not able to integrate rational functions exactly. A higher number of integration points reduces the errors for computations with the coarsest mesh to machine precision. Here the number of integration points is chosen according to (72). The convergence rates of $h$-refinement studies can be compared between computations with conforming and non-conforming meshes. The influence of the connection methods is clearly visible with the help of this example. The geometry and the boundary conditions are given in Fig. 4, where $q=10$. The wall thickness is $t=1$. A Young's modulus of $E=100$ and a Poisson ratio of $v=0.3$ are used. Computations are performed with linear and nonlinear kinematics. The initial mesh for conforming computations consists of $10 \times 2$ elements per patch, where the coarsest mesh is subdivided into ten elements in the first parametric direction and two elements in the second parametric direction. For non-conforming computations patch 2 is discretized with $10 \times 3$ elements, whereas patch 1 is not altered. The initial elements are equally subdivided in both parametric directions. Studies are performed with NURBS basis functions of degree $p=3$ and $p=4$, where the degree is elevated using $k$-refinement. 


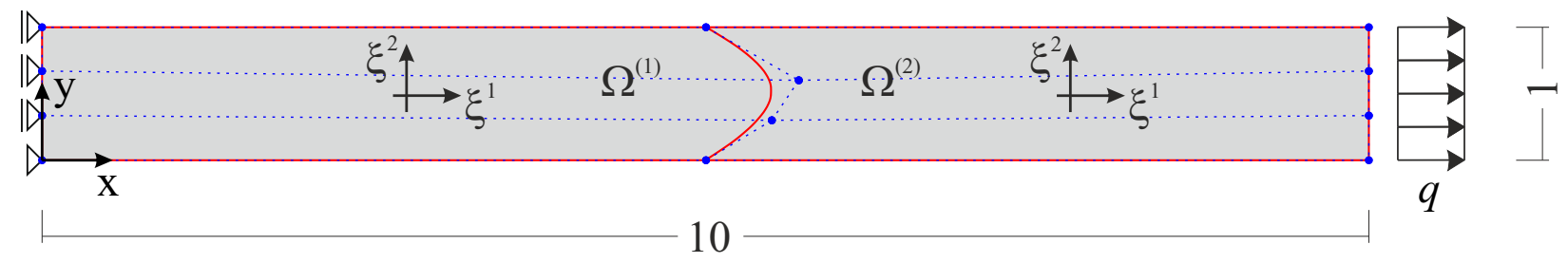

Figure 4: Beam under pure tension: Problem geometry, boundary conditions, coarsest mesh (red lines) and control point net (blue dashed lines).

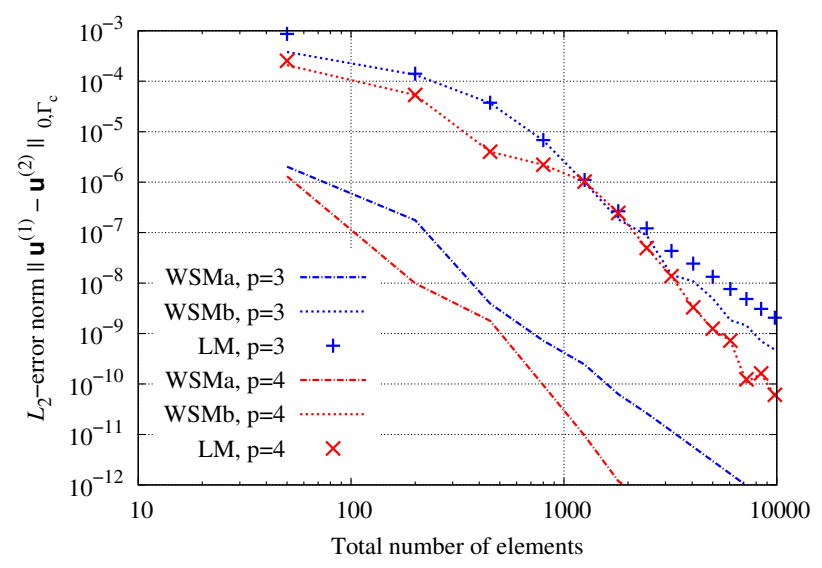

(a) Linear computations

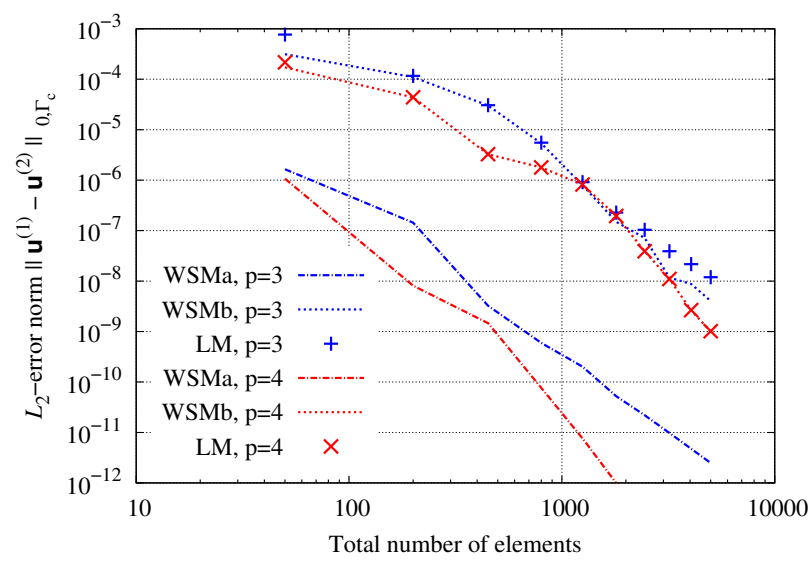

(b) Nonlinear computations

Figure 5: Beam under pure tension: $L_{2}$-error norm of the Dirichlet interface condition.

The results of linear and nonlinear computations given in Figs. 5a-7a respectively Figs. 5b-7b are very similar. The graphs start to level off at an order of $10^{-12}$ and thus this value is used as a lower bound for the diagrams. The $L_{2}$-error norms of the mutual deformations along the interface are given in Fig. 5. The variant of the weak substitution method, where the patch which has more interface control points is chosen as master patch, is denoted by WSMb. This scheme and the Lagrange multiplier method, denoted by LM, entail quite similar results. The error of the mutual deformations of the WSMa scheme, which classifies the patch with less interface control points as master patch, is several orders of magnitude smaller. The error of conforming discretizations connected by shared degrees of freedom is in the order of $10^{-14}$, reflecting numerical accuracy, and thus not displayed here. The $L_{2}$-error norms of the mutual tractions along the interface are given in Fig. 6. Due to the $C^{0}$-continuity along the interface, the conforming discretizations (denoted by conf.) also yield a Neumann interface error. This error decreases exponentially with a leveling off at approximately $10^{-11}$. The results for the non-conforming discretizations decrease at a similar slope, but with a larger error and some smaller oscillations. The WSMa scheme yields better results than the LM method and the WSMb scheme. The $L_{2}$-error norm $\left\|\mathbf{S}-\mathbf{S}^{h}\right\|_{0, \Omega}$, where $\mathbf{S}$ are the exact stresses and $\mathbf{S}^{h}$ the results of the computations, assesses the stress distribution throughout the whole domain. The impact of the connection methods on the global solution can clearly

Table 1: Control points and knot vector of the unrefined NURBS connection line with degree $p=3$.

\begin{tabular}{cc}
\hline Control point & Coordinates $(\mathrm{x}, \mathrm{y}, \mathrm{w})$ \\
\hline 1 & $(5,0,1)$ \\
2 & $(5.5,0.3,1.2)$ \\
3 & $(5.7,0.6,1.5)$ \\
4 & $(5,1,1)$ \\
\hline
\end{tabular}

knot vector: $\boldsymbol{\Xi}=[0,0,0,0,1.75,1.75,1.75,1.75]$ 


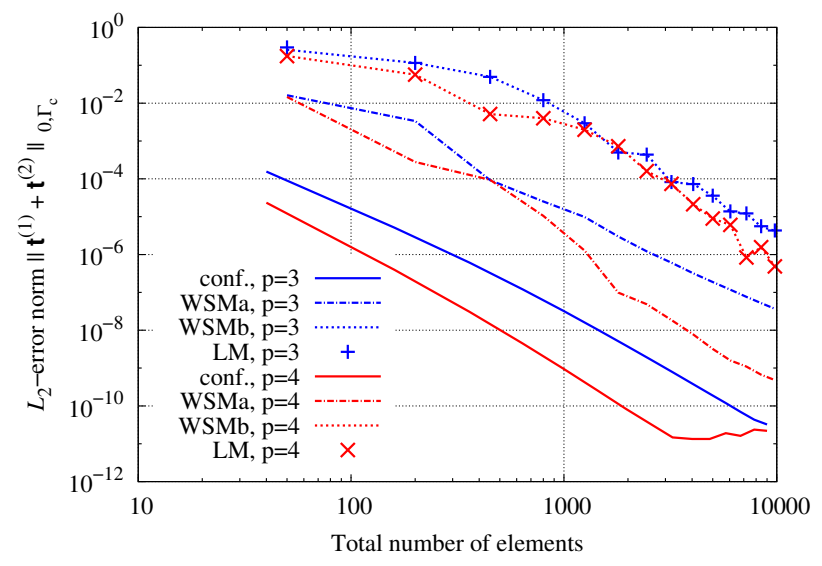

(a) Linear computations

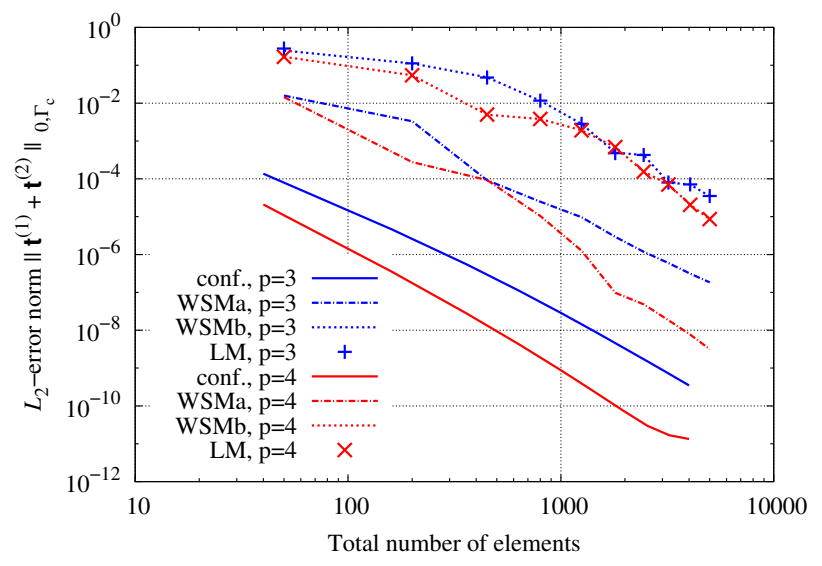

(b) Nonlinear computations

Figure 6: Beam under pure tension: $L_{2}$-error norm of the Neumann interface condition.

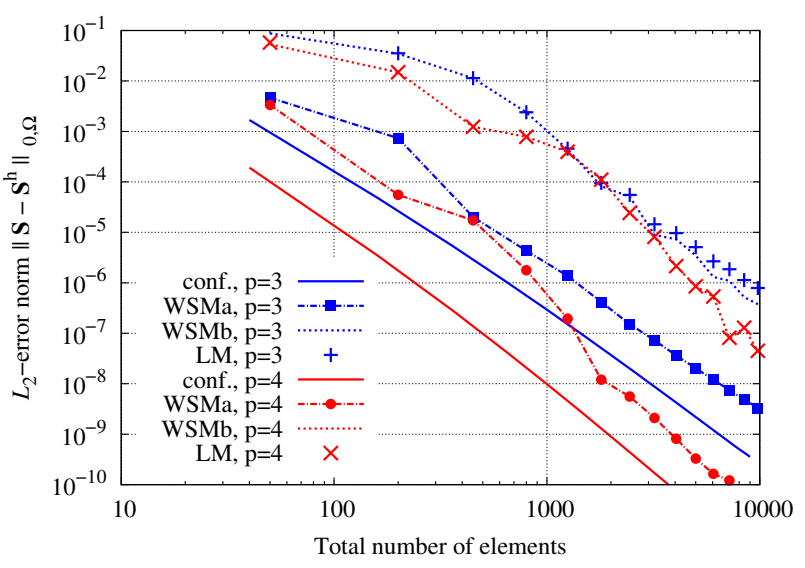

(a) Linear computations

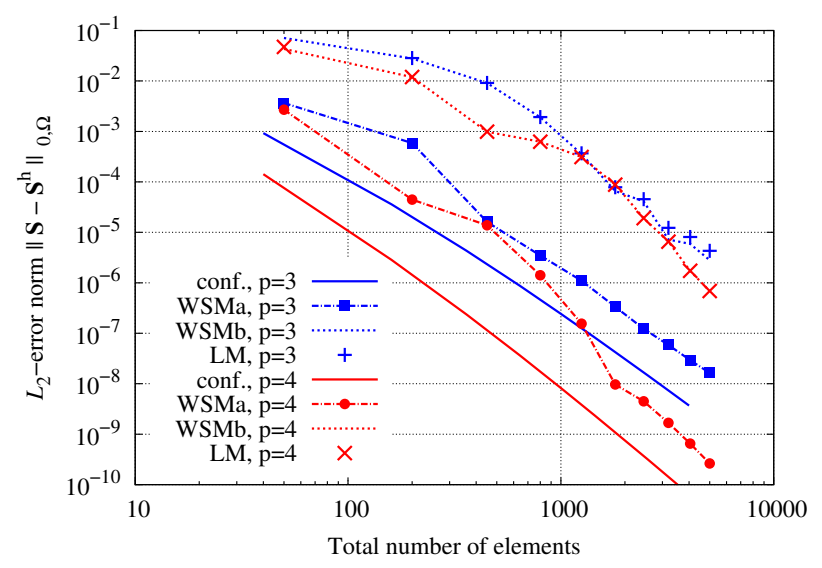

(b) Nonlinear computations

Figure 7: Beam under pure tension: $L_{2}$-error norm of the stress distribution throughout the whole domain.

be seen. The error of linear and nonlinear computations is plotted in Fig. 7. The graphs of conforming computations show exponential convergence behavior. The errors entailed by the WSMa scheme are about one order of magnitude higher than for the conforming case. The error norms of the LM method and the WSMb scheme coincide. The error of the two latter connection methods clearly has a severe impact on the global solution, as the error level is considerably higher than for conforming computations. However, it is to be noted, that all errors in this example are entailed by the connection. In more complex states of stress, the impact of the connection methods on the global solution is considerably lower, see the following examples.

\subsection{Quarter of an annulus subject to tip shear force}

The quarter of an annulus is fixed at one end and loaded with a shear force at the other end. This creates a state of stress which is dominated by in-plane bending. An analytical solution for the stress distribution is given in [17]. Geometry and boundary conditions are given in Fig. 8a. The inner and outer radius of the annular are defined by $r_{\min }=4$ and $r_{\max }=5$, respectively. Possible values of the angle $\theta$ are $0^{\circ} \leq \theta \leq 90^{\circ}$. The shear load $p=10$ has to be 


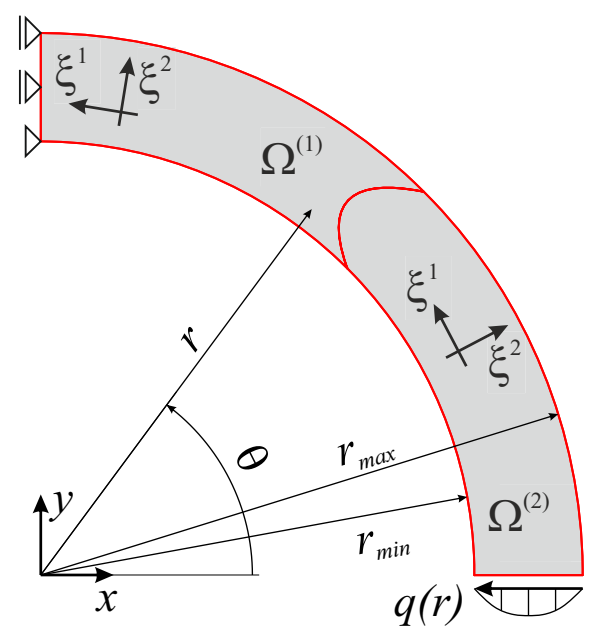

(a) Geometry

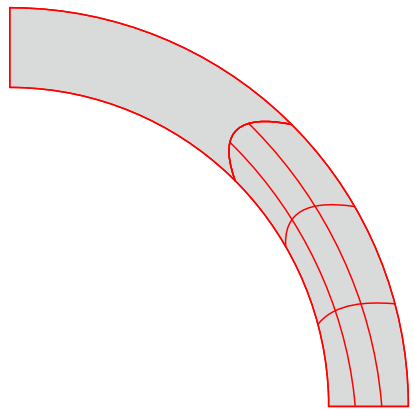

(b) $j=1$

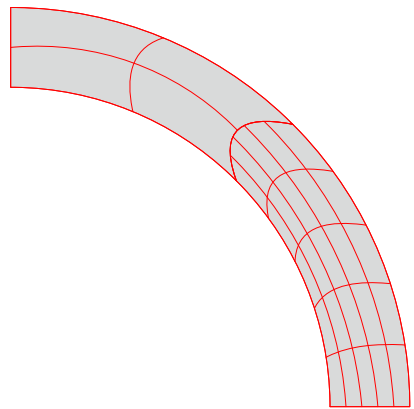

(c) $j=2$

Figure 8: Quarter of an annulus: Problem geometry, boundary conditions and the two coarsest meshes for $j: 2 j+1$ refinement.

Table 2: Control points and knot vector of the unrefined NURBS connection line with degree $p=2$.

\begin{tabular}{cc}
\hline Control point & Coordinates $(\mathrm{x}, \mathrm{y}, \mathrm{w})$ \\
\hline 1 & $(2.828427124746190,2.828427124746190,1)$ \\
2 & $(2.500000000000000,3.741657387000000,1)$ \\
3 & $(3.535533905932737,3.535533905932737,1)$ \\
\hline
\end{tabular}

knot vector: $\boldsymbol{\Xi}=[0,0,0,0.5,1,1,1]$

applied according to the analytical solution. Its distribution $q(r)$ is given by

$$
q(r)=\frac{p}{N_{0}}\left(r+\frac{r_{\min }^{2} r_{\max }^{2}}{r^{3}}-\frac{r_{\min }^{2}+r_{\max }^{2}}{r}\right)
$$

with

$$
N_{0}=r_{\text {min }}^{2}-r_{\text {max }}^{2}+\left(r_{\text {min }}^{2}+r_{\text {max }}^{2}\right) \ln \frac{r_{\text {max }}}{r_{\text {min }}} .
$$

The domain is discretized with two NURBS patches with a curved connection line, which is defined in Tab. 2 . The wall thickness is $t=1$. A Young's modulus of $E=10^{5}$ and a Poisson ratio of $v=0$ are used. Computations are performed with linear kinematics. The existence of an analytical solution allows the influence of the connection methods to be assessed with the help of the $L_{2}$-error norm $\left\|\mathbf{S}-\mathbf{S}^{h}\right\|_{0, \Omega}$. Convergence studies are provided for three different non-conforming discretizations. In the first two discretizations meshes with an element ratio $j: 2 j+1$ along the interface are used. The coarsest mesh is displayed in Fig. 8b. The number of elements in patch 1 is given by $j^{2}$, and by $(2 j+1)^{2}$ in patch 2 . The factor $j \in\{1, \ldots, 25\}$ defines the refinement strategy for $h$-refinement. The resulting mesh for $j=2$ is given in Fig. 8c. The results in Figs. 9a-11a use an equal degree $p=2$ for all parametric directions in both patches, whereas in Figs. 9b-11b the degrees $p_{1}^{(1)}=4, p_{2}^{(1)}=3, p_{1}^{(2)}=5$ and $p_{2}^{(2)}=4$ are used. The third discretization uses subdomains with an element ratio $j: j$ along the interface, and $j^{2}$ elements both in patch 1 and in patch 2. The resulting mesh for $j=3$ is given in Fig. 12a. The orders $p_{1}^{(1)}=4, p_{2}^{(1)}=4, p_{1}^{(2)}=6$ and $p_{2}^{(2)}=6$ are used in Fig. 12b.

The error of the mutual deformations of the two $j: 2 j+1$-refined discretizations is given in Fig. 9. In both cases the WSMb scheme and the LM method yield very similar results. The graphs of WSMb and LM show small oscillations in the higher order case in Fig. 9b, but for an increasing number of elements a linear trend is visible. The WSMa scheme exhibits a smaller error level than the WSMb scheme. However, the slope is equal for all methods in the fine limit. The traction interface condition is assessed in Fig. 10. All presented methods yield an traction interface 


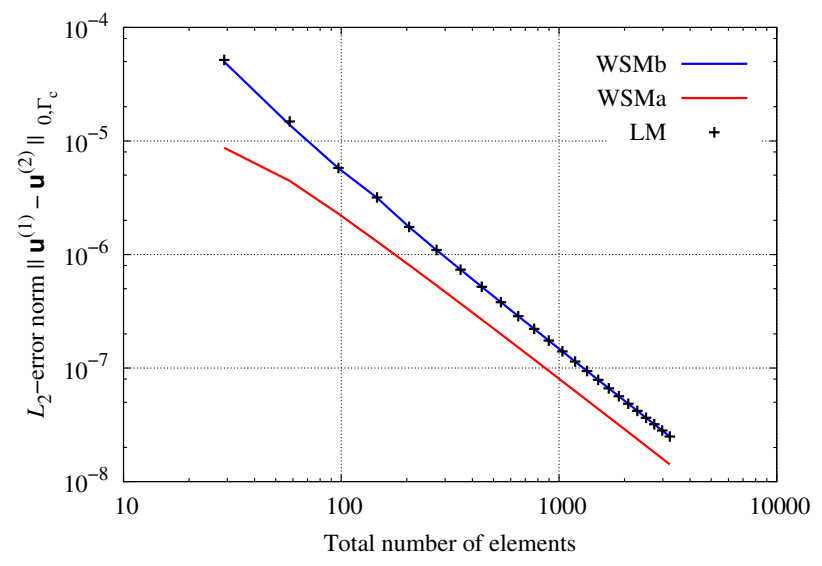

(a) $p_{1}^{(1)}=2, p_{2}^{(1)}=2, p_{1}^{(2)}=2, p_{2}^{(2)}=2$

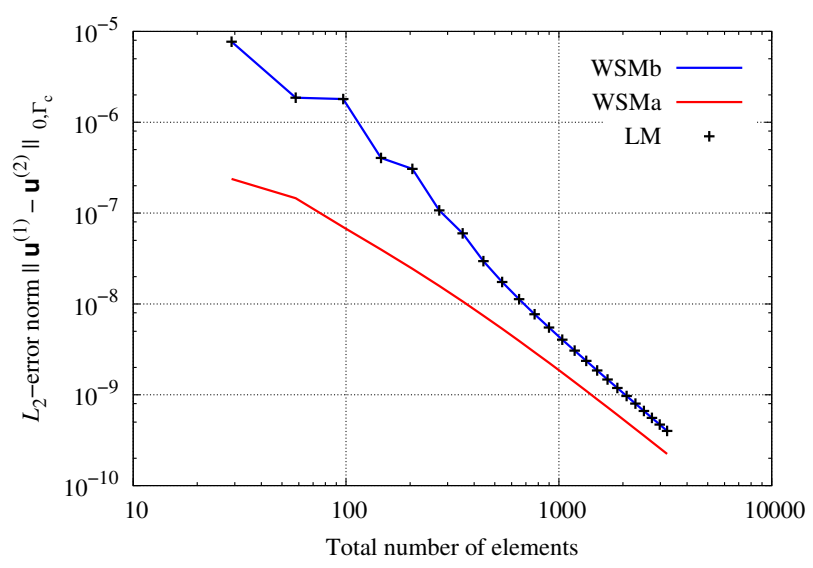

(b) $p_{1}^{(1)}=4, p_{2}^{(1)}=3, p_{1}^{(2)}=5, p_{2}^{(2)}=4$

Figure 9: Quarter of an annulus: $L_{2}$-error norm of the Dirichlet interface condition for $j: 2 j+1$ refinement.

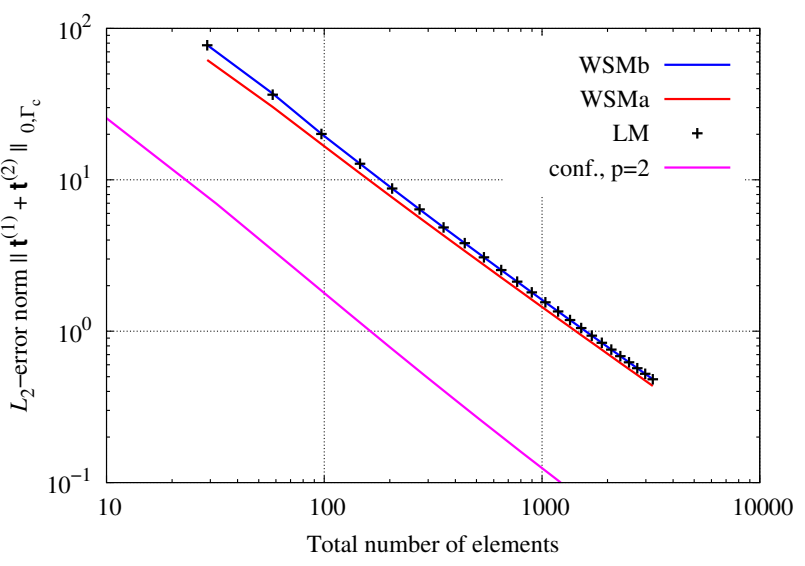

(a) $p_{1}^{(1)}=2, p_{2}^{(1)}=2, p_{1}^{(2)}=2, p_{2}^{(2)}=2$

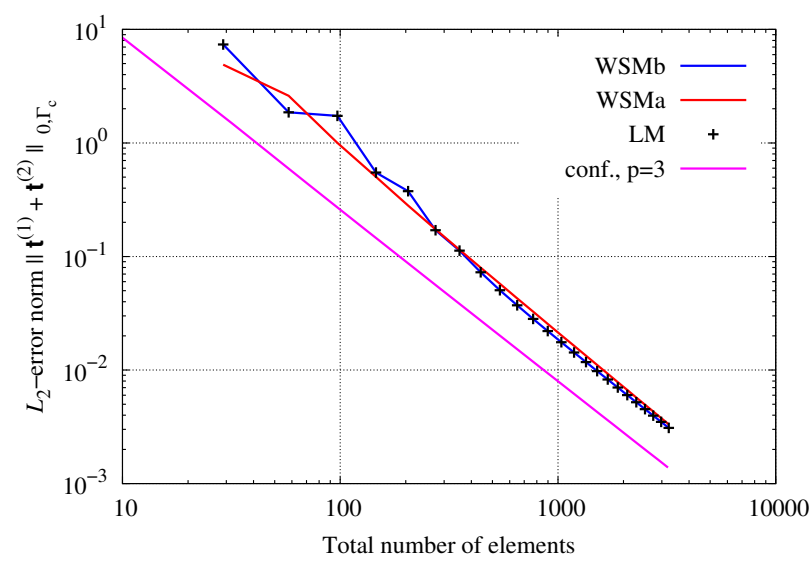

(b) $p_{1}^{(1)}=4, p_{2}^{(1)}=3, p_{1}^{(2)}=5, p_{2}^{(2)}=4$

Figure 10: Quarter of an annulus: $L_{2}$-error norm of the Neumann interface condition for $j: 2 j+1$ refinement.

error which is about one order of magnitude higher than the traction interface error for computations with conforming meshes. The results of the LM method and the WSMb scheme are not distinguishable from each other by the eye. The results of the WSMa scheme are very similar to the WSMb scheme.

The influence of the connection methods on the distribution of the stresses throughout the whole domain is the crucial point in the assessment of connection techniques. This is examined with the help of the $L_{2}$-error norm $\| \mathbf{S}-$ $\mathbf{S}^{h} \|_{0, \Omega}$ in Fig. 11. It is important to note, that unlike in the preceding figures, the number of elements within the respective subdomain is used as abscissa. Thus, the error levels of the non-conforming computations can be compared to conforming computations of the same degree within this patch. The results of the low order case in Fig. 11a are explained as follows. In patch 1 the graphs of the error levels of conforming and non-conforming computations with all three methods overlap very well. Thus, the results in the coarser meshed subdomain $\Omega^{(1)}$ are not influenced negatively by the presented connection methods. Computations with the LM method as well as with the WSMb scheme yield, beginning from 100 elements, the same error level in patch 2 as conforming computations. Thus, the global stress distribution is not significantly disturbed by the WSMb scheme and the LM method for this discretization. The stress error in patch 2 of computations with the WSMa scheme is slightly larger than in the conforming case. Thus, the WSMa scheme has a negative impact on the global stress distribution. In the higher order case in Fig. 11b no connection method has a negative impact on the error distribution in subdomain $\Omega^{(1)}$, as all graphs overlap very well. 


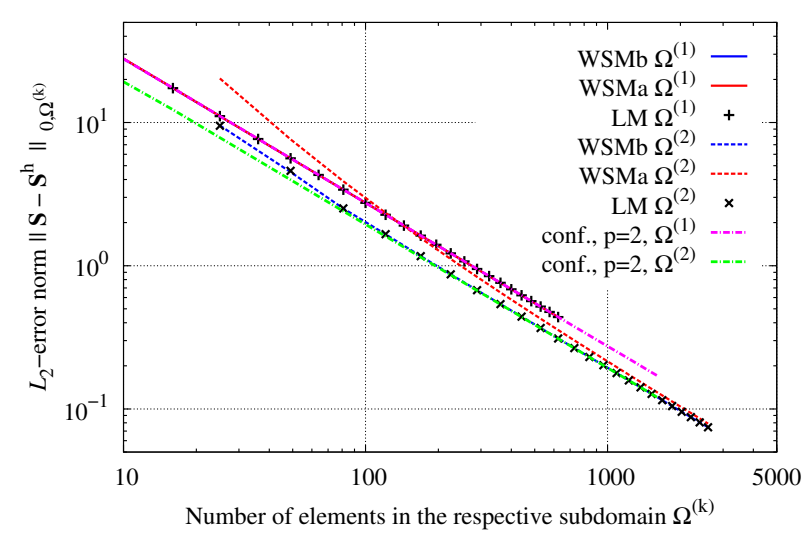

(a) $p_{1}^{(1)}=2, p_{2}^{(1)}=2, p_{1}^{(2)}=2, p_{2}^{(2)}=2$

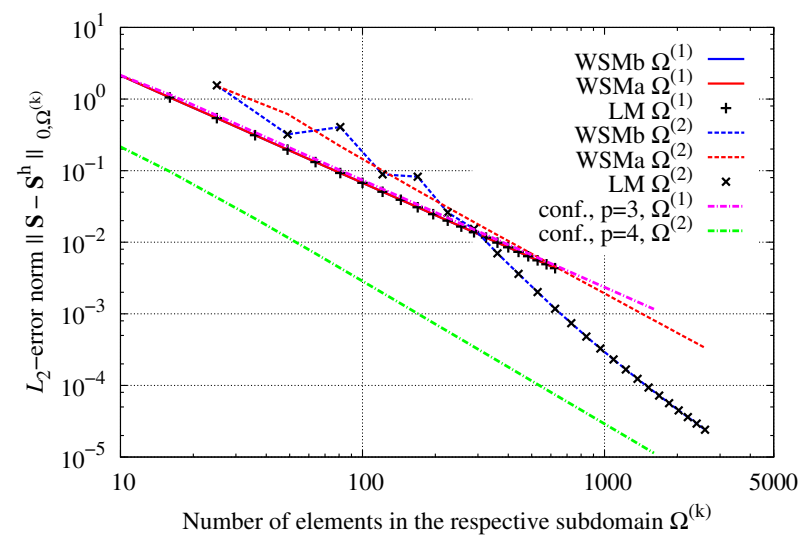

(b) $p_{1}^{(1)}=4, p_{2}^{(1)}=3, p_{1}^{(2)}=5, p_{2}^{(2)}=4$

Figure 11: Quarter of an annulus: $L_{2}$-error norm of the stress distribution throughout the whole domain for $j: 2 j+1$ refinement.

The situation is different in subdomain $\Omega^{(2)}$. The error levels of the WSMb scheme and the WSMa scheme are similar for coarse discretizations. The WSMb scheme approaches the error level of conforming computations for finer meshes, but does not reach it. In contrast to that, the error in $\Omega^{(2)}$ of the WSMa scheme almost retains the slope of the graphs for subdomain $\Omega^{(1)}$. The results of the WSMb scheme and the LM method coincide very well.

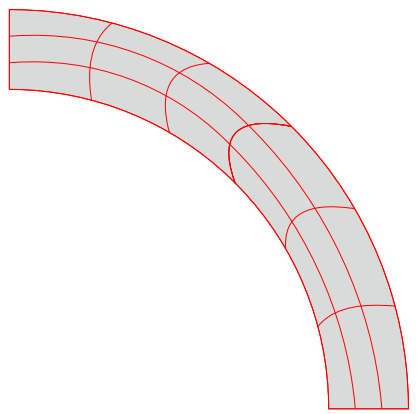

(a) Mesh for $j=3$

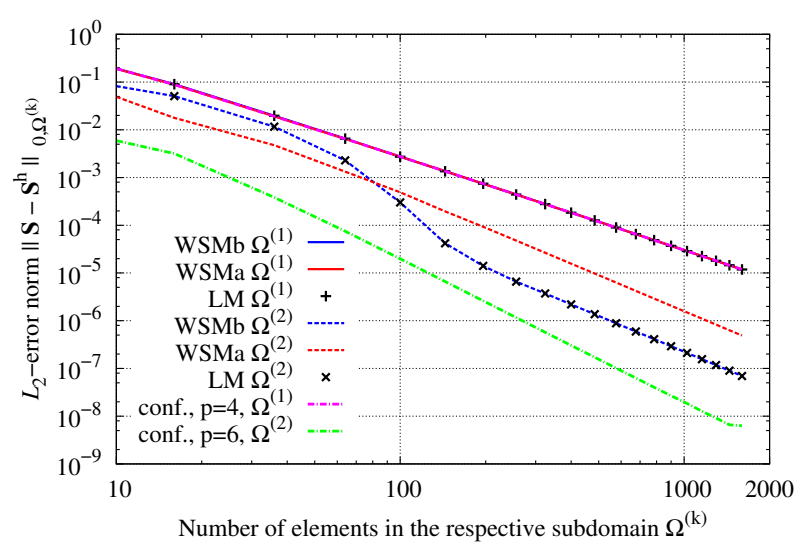

(b) $L_{2}$-error norm of the stress distribution throughout the whole domain.

Figure 12: Quarter of an annulus: Two subdomains with fitting number of elements along the interface, but differing orders: $p_{1}^{(1)}=4, p_{2}^{(1)}=4$, $p_{1}^{(2)}=6, p_{2}^{(2)}=6$.

The influence of the connection methods on the distribution of the stresses throughout the whole domain is investigated in Fig. 12b for subdomains with fitting number of elements but different orders along the interface. A mesh of this kind is given in Fig. 12a. The approximative power of the basis functions is lower in patch 1 than in patch 2 . Thus, the results are more precise in patch 2 . The results of all connection methods coincide very well with results of conforming computations in subdomain $\Omega^{(1)}$. The situation in subdomain $\Omega^{(2)}$ is similar to Fig. 11b. The LM method and the WSMb scheme overlap perfectly. For coarse meshes the WSMa scheme performs better than the WSMb scheme, but its slope stays almost constant for mesh refinement. In contrast to that, the peformance of the WSMb scheme improves beginning from around 100 elements, and the graph is closer to the conforming case.

The results of this numerical example can be summed up as follows. All presented connection methods have 
influence on the global stress distribution in the finer discretized patch (referring to more elements and higher order). The influence is not significant and decreases with mesh refinement in the case of equal orders in all subdomains. If the orders of the patches along the interface are not equal, then the approximation power of the higher order cannot be fully exploited. The accuracy of the WSMb scheme and the LM method is superior to the accuracy of the WSMa scheme.

\subsection{Elastic plate with circular hole}

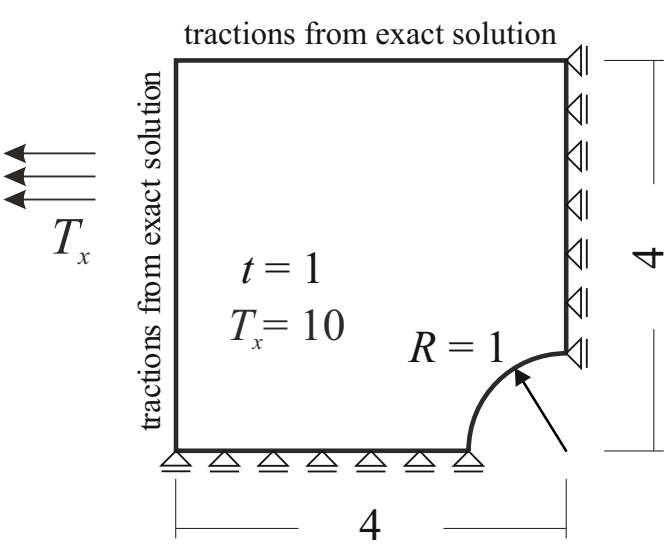

(a) Problem geometry and loading

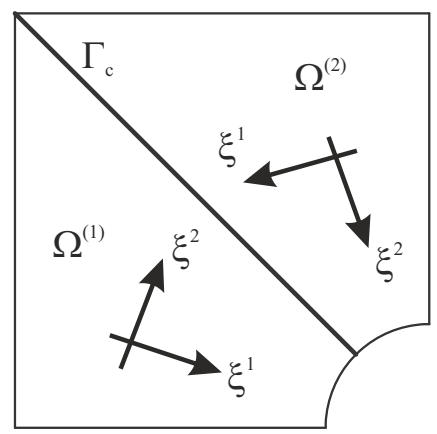

$E=1 \cdot 10^{5}, v=0$

(b) Straight interface

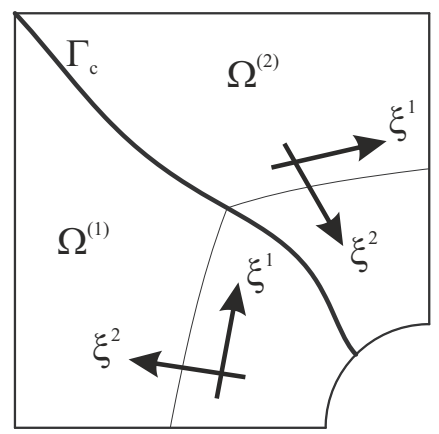

$E=1 \cdot 10^{2}, v=0.3$

(c) Curved interface

Figure 13: Elastic plate with circular hole: Problem setup, material properties and chosen subdomains for the two different computed discretization types. The parametric directions and the coarsest mesh are given.

The elastic plate with hole is a common benchmark in isogeometric analysis. The geometry can be modeled with a single NURBS patch using control points with coinciding location. For this study the domain is discretized with two regular NURBS surface patches to test the presented connection methods. The geometry and the boundary conditions are given in Fig. 13a. An analytical solution is available for an infinite plane with hole loaded with a constant traction $T_{x}$. It is given e.g. in [17], providing the analytical displacements and resulting forces. The application of the tractions from the exact solution at the free boundaries allows to use the exact solution also for the problem under consideration. Convergence studies are conducted for two different geometry models. In the first case, displayed in Fig. 13b, the interface is a straight line. In the second case the interface is curved, see Fig. 13c. The material parameters - Young's modulus $E$ and Poisson ratio $v$ - are provided in Fig. 13b and Fig. 13c for both studies.

For the convergence studies in Secs. 4.3.1-4.3.3 different degrees of NURBS basis function and refinement patterns are used. The discretization scheme for each convergence study is characterized by a combination of an uppercase letter defined in Tab. 3 and a number defined in Tab. 4. The parametric directions are given in Fig. 13b and 13c. For example, the figure caption A1 denotes that the computations are performed with refinement rule A and NURBS degree rule 1. Applied to the straight interface model, this denotes that the NURBS degree in both patches is 1 along the interface and 2 in the other parametric direction. The coarsest mesh determined with $j=1$ uses one element for patch 1 and nine elements for patch 2 .

Table 3: Refinement patterns for the elastic plate with hole.

\begin{tabular}{|c|c|c|c|}
\hline Refinement rule & Discretization: & $\begin{array}{c}\text { Subdomain } \Omega^{(1)} \\
\left(n_{1}^{(1)}-p_{1}^{(1)}\right) \times\left(n_{2}^{(1)}-p_{2}^{(1)}\right)\end{array}$ & $\begin{array}{c}\text { Subdomain } \Omega^{(2)} \\
\left(n_{1}^{(2)}-p_{1}^{(2)}\right) \times\left(n_{2}^{(2)}-p_{2}^{(2)}\right)\end{array}$ \\
\hline A & & $j \times j$ & $(2 j+1) \times(2 j+1)$ \\
\hline B & & $5 j \times 10 j$ & $5 j \times 10 j$ \\
\hline $\mathrm{C}$ & & $5 j \times 10 j$ & $5 j \times 12 j$ \\
\hline
\end{tabular}



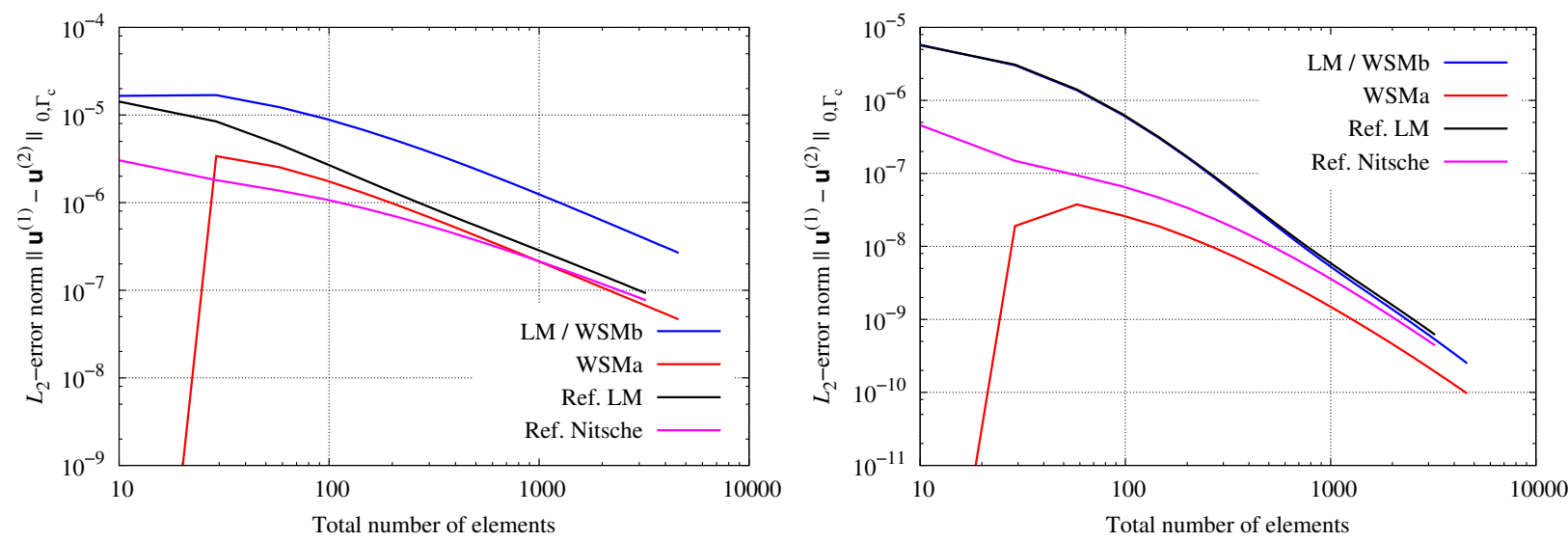

(a) Discretization scheme $\mathrm{A} 1$

$p_{1}^{(1)}=1, p_{2}^{(1)}=2, p_{1}^{(2)}=2, p_{2}^{(2)}=1$

(b) Discretization scheme A2:

$p_{1}^{(1)}=4, p_{2}^{(1)}=3, p_{1}^{(2)}=4, p_{2}^{(2)}=5$

Figure 14: $L_{2}$-error norm of the Dirichlet interface condition (mutual deformations) along the interface for the elastic plate with hole with straight interface. Reference values taken from [17].

\subsubsection{Two patches with straight connection line: Comparison to reference results}

The elastic plate with hole described by two NURBS patches with a straight interface allows a comparison to the numerical results of Apostolatos et al. [17]. Geometry, boundary conditions, discretization and material parameters are chosen accordingly to allow a precise comparison. The refinement pattern chosen in [17] of $j: 2 j+1$ along the interface produces highly irregular meshes. Computations are performed with a low degree of NURBS using discretization scheme A1 and a high degree of NURBS using scheme A2. This is in accordance with the computations reported in [17]. Results using the Lagrange multiplier method and Nitsche's method are taken from [17] and denoted by Ref. LM, respectively Ref. Nitsche in Figs. 14-16. The results of the weak substitution method using the WSMb scheme and the results from the LM method agree to a precision of $10^{-10}$ for the current example. The difference is not distinguishable by the eye. Thus, in Figs. 14-17 a single data line is used to represent the results of LM and WSMb. The deformation and stress results of the LM method do not differ for values $\eta \in\left[1 \cdot 10^{0}, 1 \cdot 10^{5}\right]$. The results in Figs. 14-17 are computed with $\eta=E=1 \cdot 10^{5}$.

The $L_{2}$-error norm of the mutual displacements of patch 1 and patch 2 along the interface $\Gamma_{c}$ is given in Fig. 14 . In both discretizations the WSMa scheme performs better than the LM/WSMb scheme. This is in accordance to the behavior observed in Sec. 4.1. The coarsest mesh, which arises for $j=1$, is a hierarchical mesh. In this case an exact substitution relation according to $[20,21]$ exists. The WSMa scheme reproduces this exact substitution relation and thus the error norm of the mutual deformations is in the range of the numerical precision. For higher values of $j$ non-conforming meshes arise, where no exact substitution is possible. Thus, a kink occurs in the graph of the WSMa scheme in Fig. 14. For the low order NURBS in Fig. 14a the graph of the reference Lagrange multiplier method data from [17] is between the values for WSMa and LM/WSMb. The deviation between the Ref. LM values and the own computations with the LM method described in Sec. 3.1 is due to differing parametrizations of the Lagrange multiplier field. In the high order case in Fig. 14b the Ref. LM and the LM/WSMb curves almost coincide. The reference Nitsche values exhibit superior behavior in comparison to the Lagrange multiplier methods. Summing up, the deformation

Table 4: Degrees of the NURBS basis functions for the elastic plate with hole.

\begin{tabular}{c|rrrr}
\hline NURBS degrees rule: & $p_{1}^{(1)}$ & $p_{2}^{(1)}$ & $p_{1}^{(2)}$ & $p_{2}^{(2)}$ \\
\hline 1 & 1 & 2 & 2 & 1 \\
2 & 4 & 3 & 4 & 5 \\
3 & 4 & 4 & 4 & 4 \\
4 & 5 & 5 & 5 & 5 \\
\hline
\end{tabular}



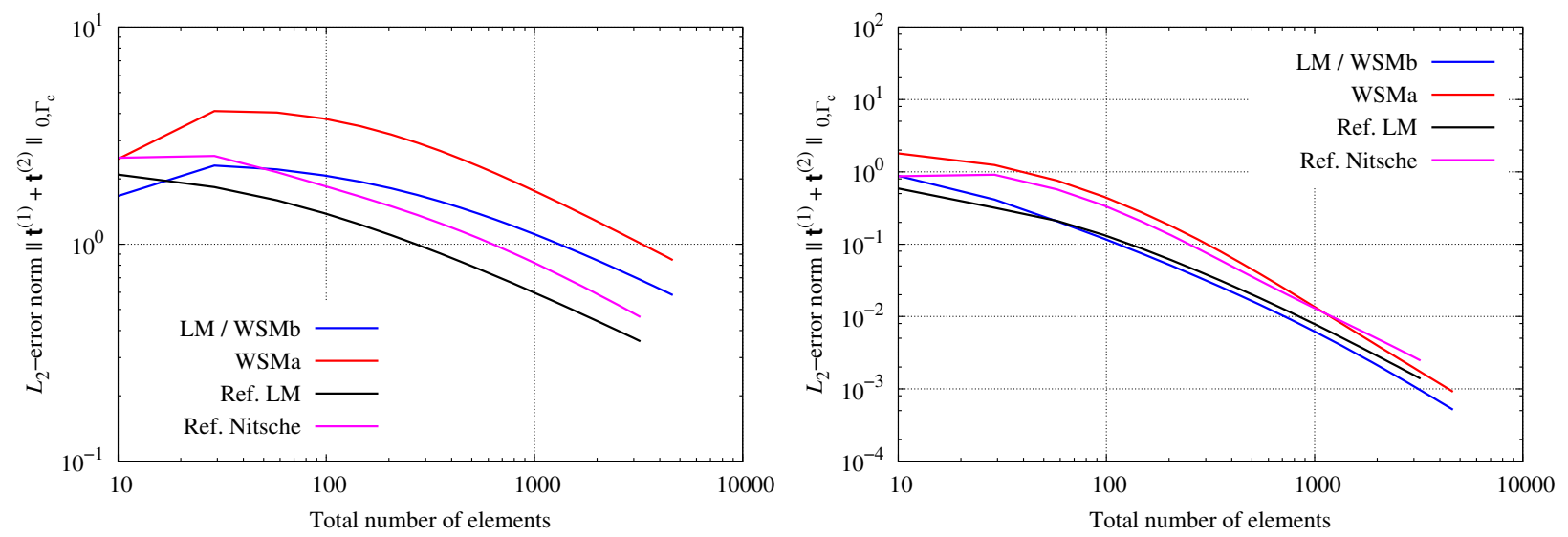

(a) Discretization scheme A1:

$p_{1}^{(1)}=1, p_{2}^{(1)}=2, p_{1}^{(2)}=2, p_{2}^{(2)}=1$

(b) Discretization scheme A2:

$p_{1}^{(1)}=4, p_{2}^{(1)}=3, p_{1}^{(2)}=4, p_{2}^{(2)}=5$

Figure 15: $L_{2}$-error norm of the Neumann interface condition along the interface for the elastic plate with hole with straight interface. Reference values taken from [17].

error norm converges with almost the same slope for all connection methods with a very small error level. The error of the interface tractions is displayed in Fig. 15. All considered connection methods perform comparable. The slopes and error levels are similar. The Ref. LM values are the most accurate in the low degree NURBS discretization case in Fig. 15a, whereas the LM/WSMb values are the most accurate in Fig. 15b. It is to be remarked, that the traction interface error of the WSMb scheme is in all calculations smaller than the error of the WSMa scheme. The numerical interface tractions are compared to the exact interface tractions in Fig. 16, separately for each side of the interface. The LM/WSMb scheme outperforms the WSMa scheme in the low degree case in Fig. 16a. The reference Lagrange multiplier results are more accurate as the own LM computations for fine meshes and have a larger error for coarse meshes. This is due to the different parametrizations of the Lagrange multiplier field. In the high order case in Fig. 16b the slope of all methods is approximately the same. The lowest error level is attained with the LM/WSMb scheme in patch 2, followed by the Ref. LM values in patch 1 . This shows the strong dependance of the Lagrange multiplier method on the parametrization of the Lagrange multiplier field.

The impact of the connection methods on the global solution is assessed with the $L_{2}$-error norm of the stresses over the whole domain. Results are given in Fig. 17 for each subdomain $\Omega^{(k)}$ and for the complete domain $\Omega$. The error of computations with the WSMa scheme is slightly higher than of those using the WSMb scheme, respectively the LM scheme. The WSMa scheme yields a lower convergence rate for patch 2 than the WSMb scheme for the discretization scheme A2 displayed in Fig. 17b. The slope of the WSMa graph for patch 2 is approximately the same as for patch 1, despite the higher approximation power in patch 2, which is entailed by the higher order of the basis functions. The WSMa scheme yields a lower number of degrees of freedom along the interface than WSMb. Thus, despite the more accurate connection of deformations (see Fig. 14b), the lower number of degrees of freedom along the interface slightly impairs the global stress convergence behavior as well as the Neumann interface condition (see Fig. 15b). The WSMb scheme, which uses the set of interface control points with the highest cardinality as master set, is able to describe the stresses more accurately.

\subsubsection{Two patches with curved connection line: linear computations}

The behavior of the two presented methods for a complex state of stress at a curved interface is shown with the help of the elastic plate with hole using two patches with a curved interface. The initial NURBS model is shown in Fig. $13 \mathrm{c}$ including material parameters and the parametric directions. Geometry and boundary conditions are chosen according to Fig. 13a. The interface curve is specified in Tab. 5. Computations are performed with linear kinematics and NURBS basis functions of degree $p=4$. For this case no reference values exist. A comparison is drawn between results with the Lagrange multiplier method and the weak substitution method. The discretization scheme $\mathrm{C} 3$ entails a ratio of $10 j: 12 j$ elements along the interface. This allows a comparison to conforming computations 

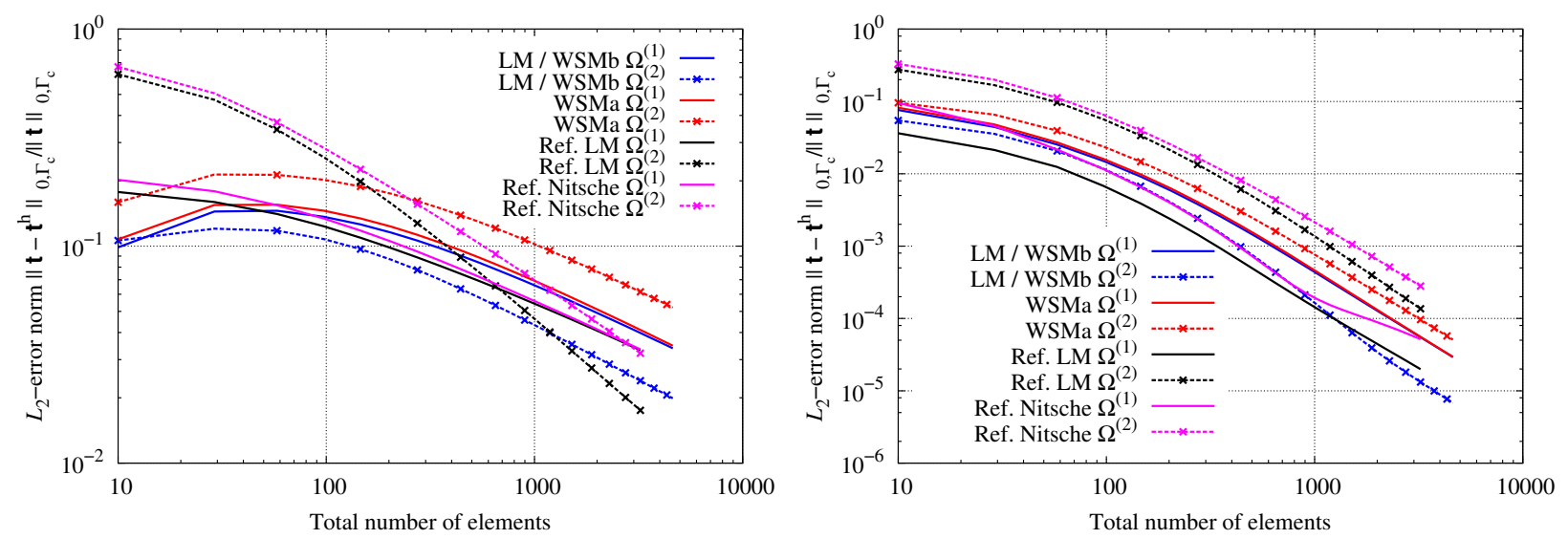

(a) Discretization scheme A1

$p_{1}^{(1)}=1, p_{2}^{(1)}=2, p_{1}^{(2)}=2, p_{2}^{(2)}=1$

(b) Discretization scheme A2:

$p_{1}^{(1)}=4, p_{2}^{(1)}=3, p_{1}^{(2)}=4, p_{2}^{(2)}=5$

Figure 16: $L_{2}$-error norm of the interface tractions along the interface for the elastic plate with hole with straight interface. Reference values taken from [17].

with discretization scheme B3 ( $10 j: 10 j$ elements along the interface). The multiplicative parameter $\eta=E=100$ is used for the computations with the LM method.

Table 5: Control points and knot vector of the unrefined NURBS connection line with degree $p=4$.

\begin{tabular}{cc}
\hline Control point & Coordinates $(\mathrm{x}, \mathrm{y}, \mathrm{w})$ \\
\hline 1 & $(-1 / \sqrt{2}, 1 / \sqrt{2}, 1)$ \\
2 & $(-1,1,1)$ \\
3 & $(-0.9,1.8,2)$ \\
4 & $(-2.9,2.4,2)$ \\
5 & $(-3.5,3.5,1)$ \\
6 & $(-4,4,1)$ \\
\hline
\end{tabular}

knot vector: $\boldsymbol{\Xi}=[-5.2686,-5.2686,-5.2686,-5.2686,-5.2686,-2.6343,0,0,0,0,0]$

Results are given in Fig. 18. The $L_{2}$-error norm of the Dirichlet interface condition has almost the same slope for the WSMa and WSMb scheme, see Fig. 18a. The error level of WSMa is slightly lower, akin to the prior examples. The LM results and the results for the WSMb scheme match very well. The Neumann interface condition in Fig. 18b reveals a similar picture. The slope of all computations is equal, but here WSMb is slightly more accurate than WSMa. The higher number of degrees of freedom along the interface entails a more accurate approximation of stresses for the WSMb scheme, whereas the WSMa scheme constrains the mutual deformations more accurately, see Sec. 4.3.1. The interface traction error of computations of conforming meshes with direct connection (DC) is around one order of magnitude lower than in the non-conforming case. In Figs. 18c and 18d the interface tractions of patch 1 respectively patch 2 are compared to the analytical solution. Here all methods perform equally well with very small differences. The comparison between Fig. 18b and Figs. 18c-18d reveals, that the conforming computations are superior in comparison to the non-conforming computations in terms of fulfilling the Neumann interface condition, but not in terms of deviation from the exact solution. The error norm of the stresses over the whole domain is given in Fig. 18e. All three presented connection methods coincide perfectly. The graph of the computations with conforming meshes (DC) is not distinguishable by the eye from the non-conforming graphs. Thus, it is to be noted, that the global solution is not impaired by the usage of non-conforming meshes in combination with the presented patch coupling methods for this example. The employed element ratio of $10 j: 12 j$ is a realistic scenario for patches with a common edge but non-conforming parametrization. As long as the interface conditions are of no further interest, which in general is the case as the integration points for the global stiffness matrix do not lie on the interface, global results do not deteriorate by allowing non-conforming meshes. 

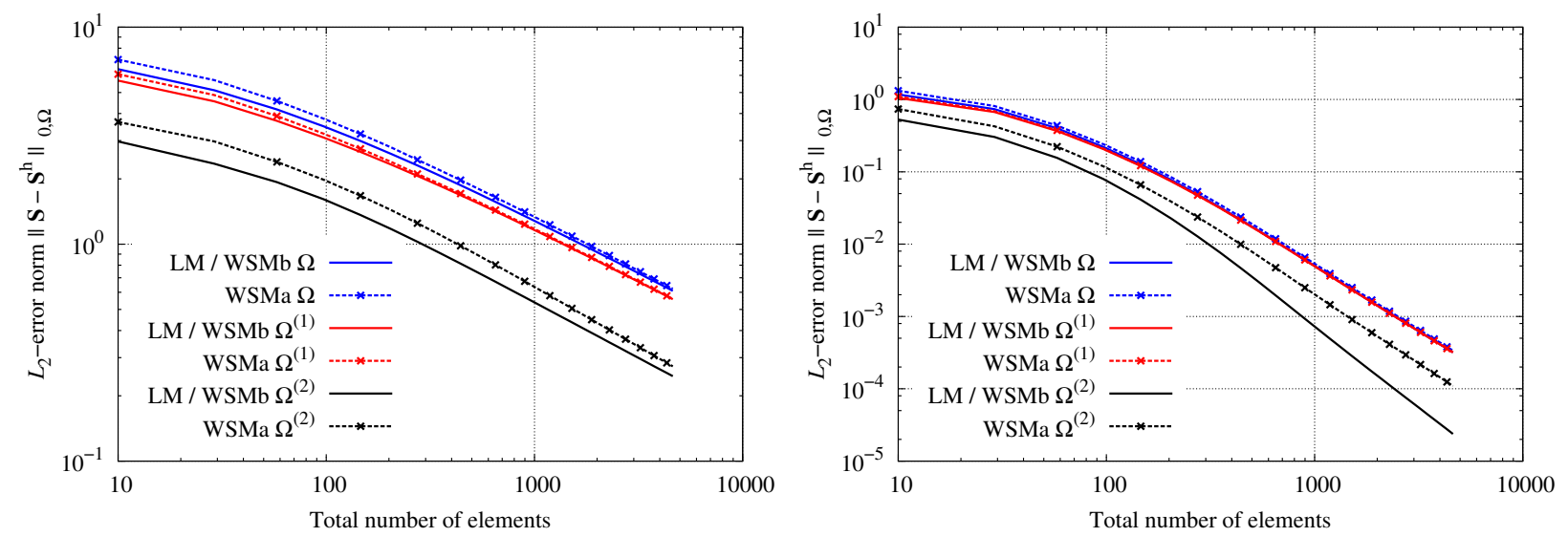

(a) Discretization scheme A1:

$p_{1}^{(1)}=1, p_{2}^{(1)}=2, p_{1}^{(2)}=2, p_{2}^{(2)}=1$

(b) Discretization scheme A2:

$p_{1}^{(1)}=4, p_{2}^{(1)}=3, p_{1}^{(2)}=4, p_{2}^{(2)}=5$

Figure 17: $L_{2}$-error norm of the stress distribution throughout the whole domain for the elastic plate with hole with straight interface.

The condition number of the stiffness matrix influences the accuracy of direct solvers and the rate of convergence of iterative solvers, see [28]. It is given in Fig. $18 \mathrm{f}$ for all presented connection methods. The condition number is determined in MATLAB with the function cond (). Both WSM schemes do not deteriorate the stiffness matrix in comparison to a direct connection using shared degrees of freedom. The condition number entailed by the LM method depends on the choice of the multiplicative parameter $\eta$. The choice $\eta=E$ increases the condition number about one order of magnitude in comparison to the direct connection for this example. The global system of equations is solved with the MATLAB routine mldivide(), which yielded the same numerical results for all examined choices of $\eta$.

\subsubsection{Two patches with curved connection line: nonlinear computations}

This example is used to test the presented connection methods in the nonlinear case for a complex state of stress. The geometry is given in Fig. 19a. The tractions from the exact solution are replaced by a constant line load $q=10$ on the left edge whereas the upper edge is not loaded. The geometry is modeled with a curved interface as displayed in Fig. 13c. The Young's modulus is $E=100$ and Poisson's ratio is $v=0.3$. Computations are performed with degree $p=4$ and $p=5$ (NURBS degree rule 3 resp. 4, see Tab. 4). Mesh refinement rule C (see Tab. 3) is used to produce non-conforming meshes. This allows a comparison to conforming computations using mesh refinement rule B. Figs. 19b and 19c provide the first two meshes arising from discretization scheme C3. The multiplicative parameter $\eta=E=100$ is used for the LM computations. In the non-conforming case, the mesh is chosen to be finer in patch 2 as the gradient of the stresses is anticipated to be larger in this patch. The error level is related to the total number of elements. Thus, the error level yielded by conforming patches should be slightly higher than in the non-conforming case. A Newton-Raphson iteration is used to solve the global system of equations. The equilibrium

Table 6: Elastic plate with hole with curved interface: nonlinear computations: Equilibrium path of the nonlinear Newton-Raphson iteration. All computations are performed for a refinement factor $j=2$ and a degree $p=4$ in both parametric directions in all subdomains.

\begin{tabular}{c|c|c|c}
\hline \multicolumn{4}{c}{ Norm of the residuum vector } \\
\hline DC, B3 (conf.) & LM, C3 & WSMa, C3 & WSMb, C3 \\
\hline $1.1593 \mathrm{E}+01$ & $1.1593 \mathrm{E}+01$ & $1.1593 \mathrm{E}+01$ & $1.1592 \mathrm{E}+01$ \\
$2.4969 \mathrm{E}+00$ & $4.4199 \mathrm{E}+00$ & $2.5006 \mathrm{E}+00$ & $2.5005 \mathrm{E}+00$ \\
$1.7423 \mathrm{E}-01$ & $2.7868 \mathrm{E}-01$ & $1.7540 \mathrm{E}-01$ & $1.7539 \mathrm{E}-01$ \\
$3.0345 \mathrm{E}-03$ & $3.7148 \mathrm{E}-03$ & $3.1076 \mathrm{E}-03$ & $3.1079 \mathrm{E}-03$ \\
$8.0205 \mathrm{E}-07$ & $8.6037 \mathrm{E}-07$ & $8.4924 \mathrm{E}-07$ & $8.4939 \mathrm{E}-07$ \\
$1.2441 \mathrm{E}-12$ & $1.2358 \mathrm{E}-12$ & $1.5593 \mathrm{E}-12$ & $1.5259 \mathrm{E}-12$ \\
\hline
\end{tabular}

path of all three connection methods is compared in Tab. 6 to conforming computations. All other discretizations 


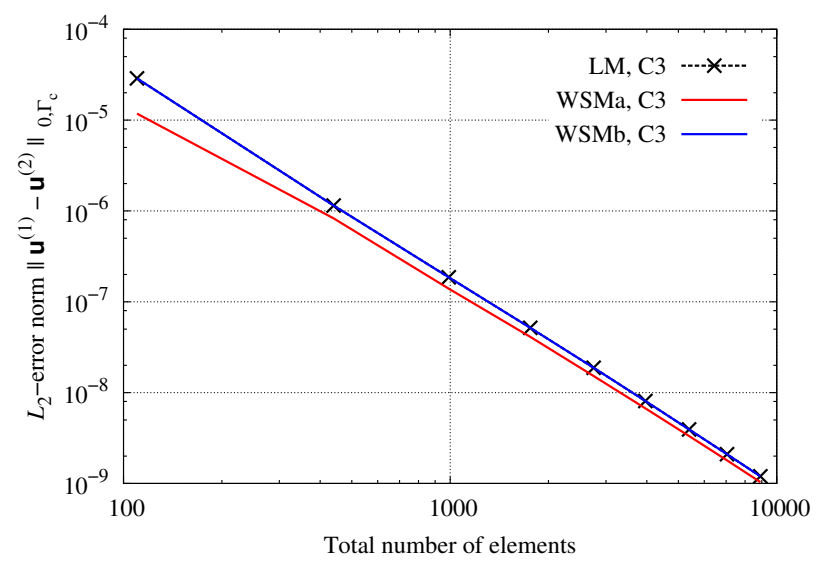

(a) Error norm of the Dirichlet interface condition.

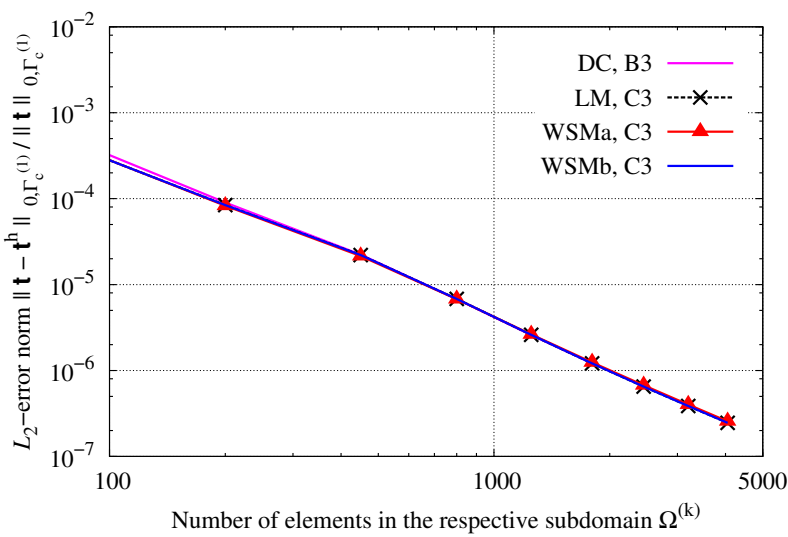

(c) Error norm of the interface tractions in patch 1.

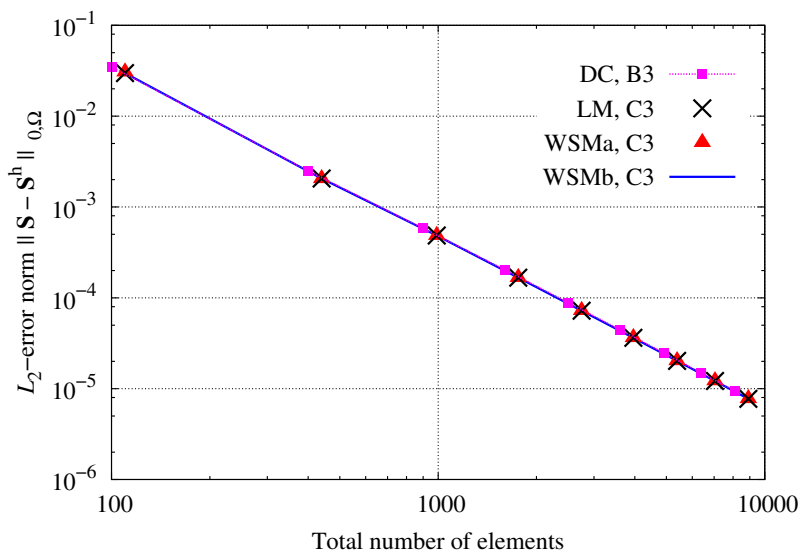

(e) Error norm of the stress distribution throughout the whole domain.

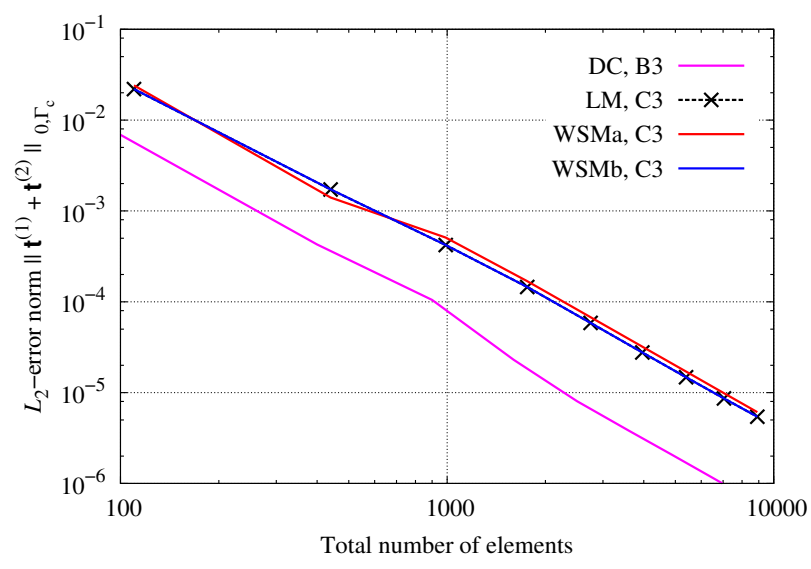

(b) Error norm of the Neumann interface condition.

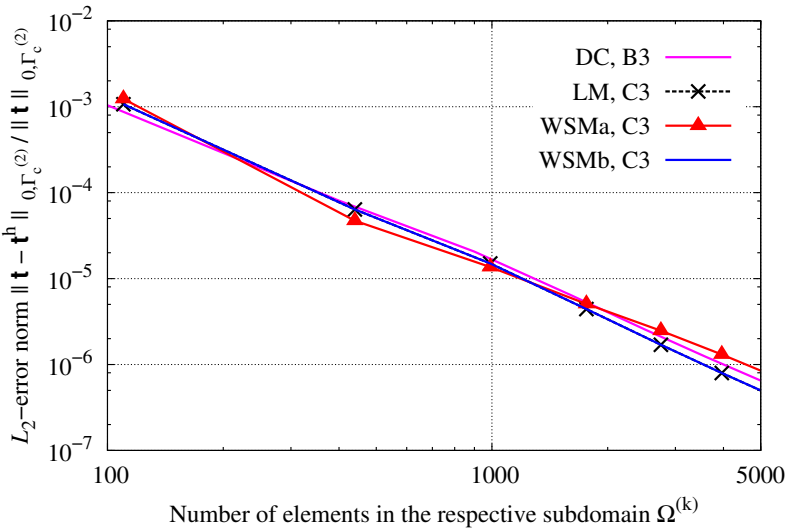

(d) Error norm of the interface tractions in patch 2.

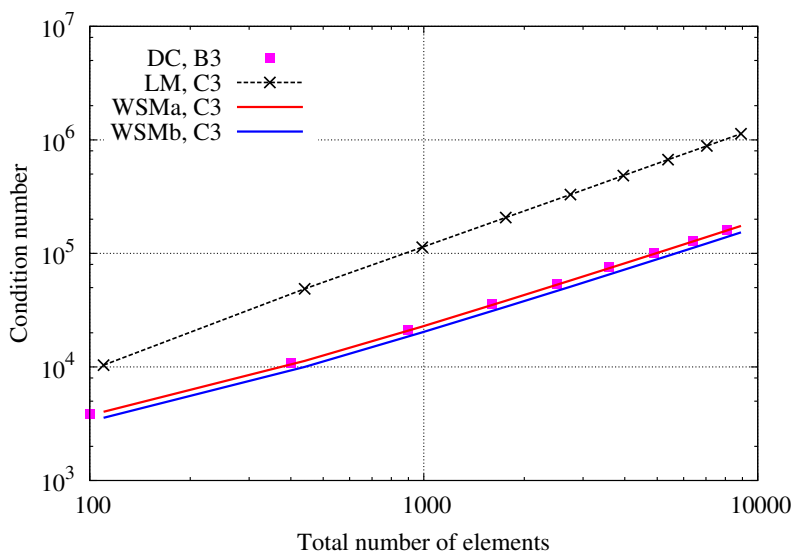

(f) Condition number of the stiffness matrix.

Figure 18: Elastic plate with hole with curved interface: linear computations: $L_{2}$-error norms and condition number for discretization schemes B3 (conforming) and C3 (non-conforming): The degree of the basis functions in both parametric directions is $p=4$ for both patches. 


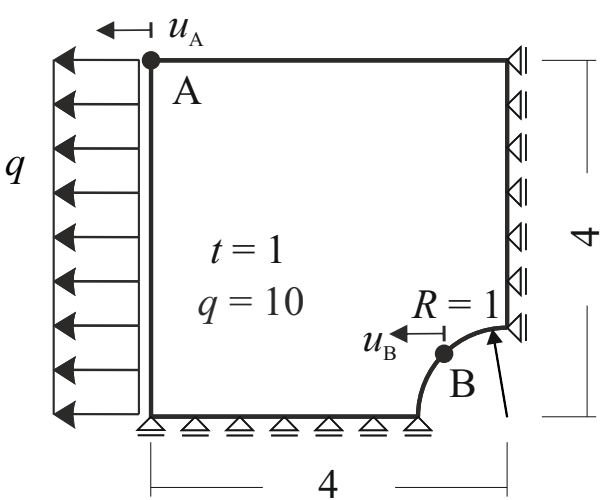

(a) Problem geometry and loading

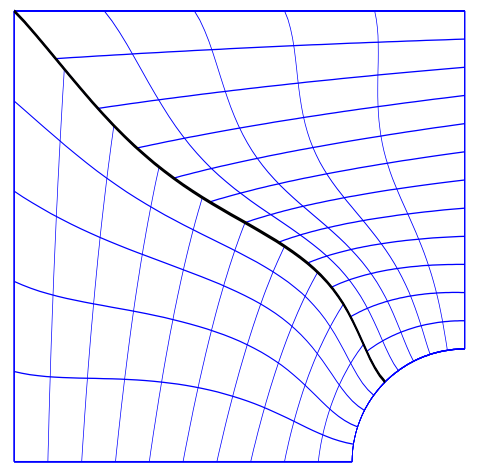

(b) Mesh for $j=1$

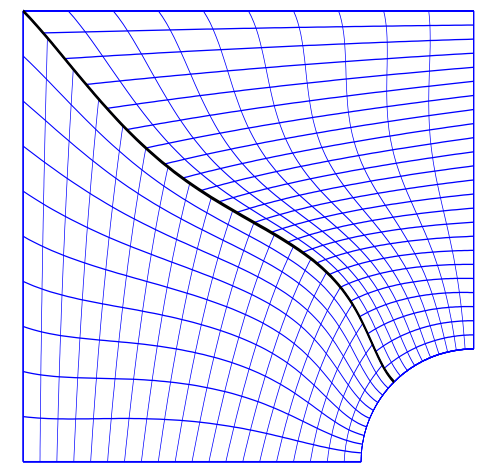

(c) Mesh for $j=2$

Figure 19: Elastic plate with hole with curved interface: nonlinear computations: Problem setup and meshes for $j=1$ and $j=2$.

showed similar convergence behavior in the nonlinear iteration. None of the methods has negative influence on the iteration behavior in all shown examples.

Results of computations with nonlinear kinematics are provided in Fig. 20. The $L_{2}$-error norms of the Dirichlet interface condition are given in Fig. 20a. Results of the WSMb scheme and the LM method coincide very well. The WSMa scheme yields errors approximately one order of magnitude smaller than the WSMb scheme. The $L_{2}$-error norms of the Neumann interface condition in Fig. 20b reveal a similar picture. The WSMa scheme yields interface errors which are in the same range than the interface errors in conforming computations (labeled DC). The errors of the WSMb scheme have a slightly higher error level, but their slope is akin. They coincide very well with the LM computations. In the nonlinear case no analytical solution exists. Thus, the energetic norm $L_{E}=\left\|\mathbf{S}^{h}: \mathbf{E}^{h}\right\|_{0, \Omega}$ is used to asses the impact of the connection methods on the global convergence behavior. The value is compared to the reference value $L_{E}^{\text {ref }}=3.896722804022$ computed with $150 \times 150$ elements of $6^{\text {th }}$ order. The relative error is plotted in Fig. 20c. The graphs of all presented methods are in very good accordance to the graphs of the respective conforming computations for both considered orders. The errors of the WSMb scheme and the LM method are slightly lower than the error of the conforming computations. The finer mesh in patch 2 yields more precise results. The error of the WSMa scheme is slightly larger than in the conforming case. Thus, the WSMa scheme slightly impaires the global solution. In addition to the error of the energetic norm, which is a measure for the behavior throughout the whole domain, discrete deformations $u_{\mathrm{A}}$ and $u_{\mathrm{B}}$ in the endpoints of the interface are examined in Fig. 21. The location of the points A and B is sketched in Fig. 19a. Points at the interface are chosen for the convergence study as the influence of the connection methods has its maximum at the interface. The deformation error is computed with respect to the reference values $u_{\mathrm{A}}=0.342816274990393$ and $u_{\mathrm{B}}=0.189249831872346$, which were attained with $200 \times 200$ elements of $6^{\text {th }}$ order in FEAP using the shell formulation [23]. Alternative computations with a finite shell element with linear basis functions [29] in FEAP converge against the same value. A mesh of $400 \times 400$ elements yielded $u_{\mathrm{A}}^{F E}=0.342812$ and $u_{\mathrm{B}}^{F E}=0.189085$. Finer discretizations were not possible due to a lack of memory. The deformation error in point A is given in Fig. 21a. The error level of the WSMb scheme and the LM method is lower than in the conforming case for both displayed degrees. Thus, the finer mesh in patch 2 yields more precise results. The deformation results are not significantly impaired by these two methods. The error level of the WSMa scheme is slightly larger than the error level in the conforming case. Thus, the WSMa scheme slightly impairs the deformation behavior in this point. In point B the situation is different. The error levels of all methods are considerably lower than in point $\mathrm{A}$, but the behavior of the connection methods cannot be classified as simple as in point A. The kinks in the graphs are due to a change from overestimated to underestimated results. Conforming computations yield the lowest error levels. The error levels of the WSMb scheme and the LM method are about one order of magnitude larger than in the conforming case. The error entailed by the WSMa scheme tends to be larger than the error of the WSMb scheme. In point B all connection methods affect the deformation results negatively. But the error is very small, and the influence is locally confined. The error of the energetic norm, which is a measure for the results throughout the entire domain is not impaired significantly. Summing up, the presented WSMb scheme and the LM method do not 


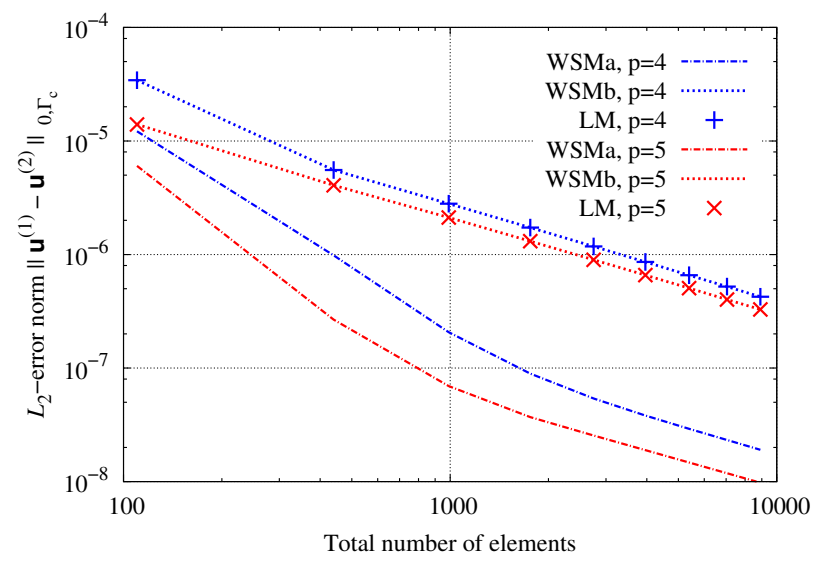

(a) Error norm of the Dirichlet interface condition.

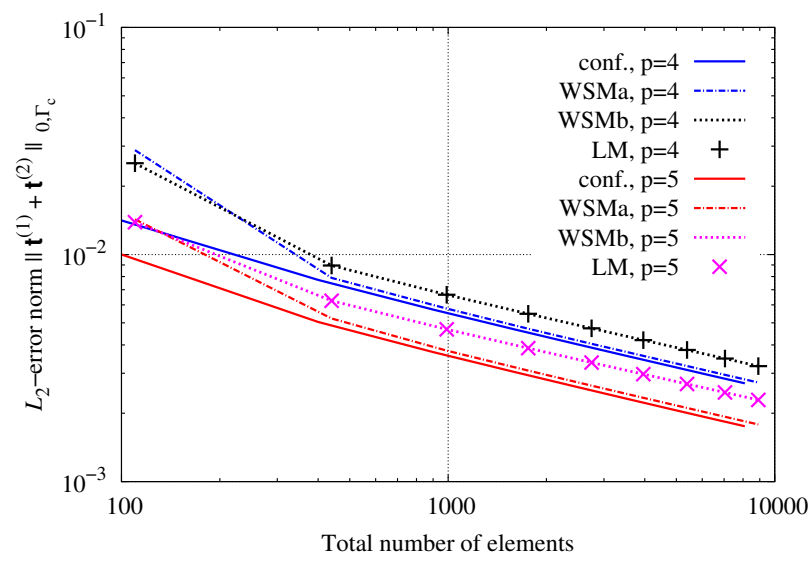

(b) Error norm of the Neumann interface condition.

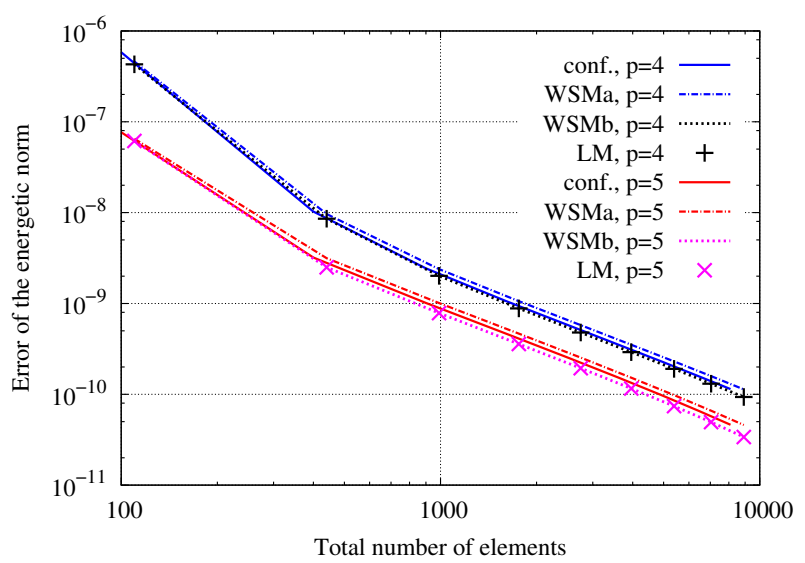

(c) Error of the energetic norm.

Figure 20: Elastic plate with hole with curved interface: nonlinear computations: $L_{2}$-error norms for discretization schemes B3, C3, B4 and C4. The conforming meshes (B3 and B4) are connected by shared degrees of freedom (DC). The non-conforming meshes (C3 and C4) are computed with the presented connection methods (WSMa, WSMb and LM).

impair the global solution significantly. The WSMa scheme has a slightly negative impact on the global solution.

\section{Conclusion and outlook}

The coupling of non-conforming subdomains is an issue especially important in NURBS-based isogeometric analysis. A new method for the connection of non-conforming NURBS patches, which is based on the mortar method, has been presented. The derivation of the method is given in detail. Several numerical examples have shown the high accuracy and robustness of the new method. Two different choices for the master/slave classification have been investigated. Depending on this choice the results vary slightly. In the most cases the WSMb scheme yields results superior to the WSMa scheme, especially regarding the influence on the global stress distribution. No final decision on the best choice is made here. Other choices for the parametrization of the interface tractions might also be a viable alternative. Future work might find a generally valid choice. To allow a comparison to existing methods for all numerical examples, a Lagrange multiplier method has been presented and implemented. The proposed weak substitution method performed as good as the Lagrange multiplier method in all examples. Convergence with mesh refinement has been shown for all examples. The decisive advantages of the new method are:

- The variational formulation is not altered. The boundary value problem is not turned into a saddle point problem. 


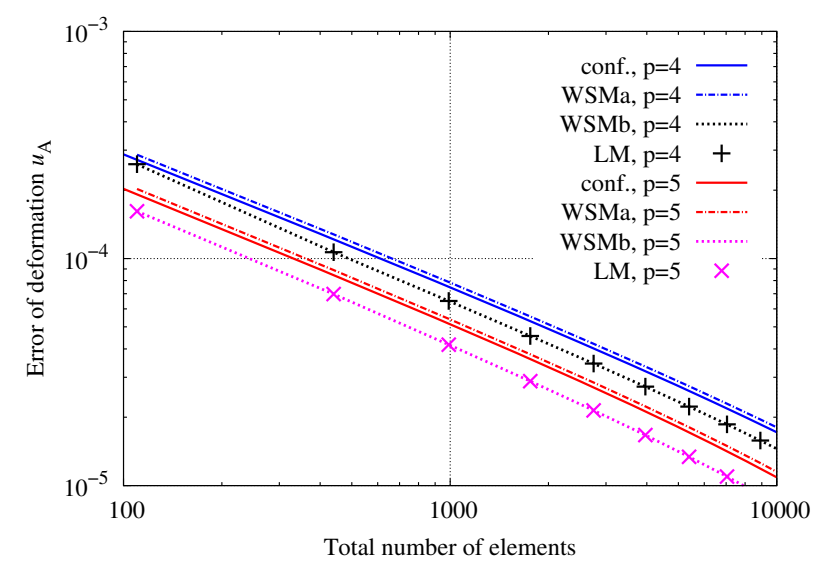

(a) Point $u_{\mathrm{A}}$.

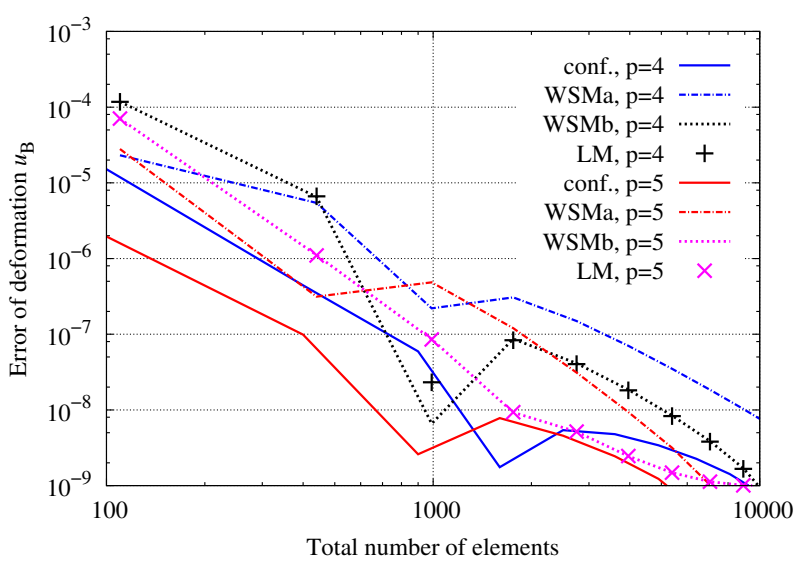

(b) Point $u_{\mathrm{B}}$.

Figure 21: Elastic plate with hole with curved interface: nonlinear computations: Error of deformation at both endpoints of the connection line for discretization schemes B3, C3, B4 and C4. The conforming meshes (B3 and B4) are connected by shared degrees of freedom (DC). The non-conforming meshes (C3 and $\mathrm{C} 4$ ) are computed with the presented connection methods (WSMa, WSMb and LM).

Thus, the global stiffness matrix remains positive definite. The number of degrees of freedom is reduced in comparison to the uncoupled problem.

- The matrices required for the coupling of subdomains need to be calculated only once as a preprocess with very low numerical effort. The method works for nonlinear computations without further measures. The computed relation matrix does not change within nonlinear iterations.

- The implementation of the method only requires changes in the assembly process of the global stiffness matrix and the residual vector. Adaptions on element level are not necessary.

- The numerical results are comparable to computations with the Lagrange multiplier method or Nitsche's method, whereas due to their nature, the two latter methods require more numerical and implementational effort.

- The condition of the global stiffness matrix does not deteriorate in comparison to conforming computations.

Summing up, the proposed weak substitution method is a simple and efficient method to couple non-conforming NURBS patches. An application of the method for curved shell elements seems feasible without further considerations. Future work might be concerned with an extension to intersections of multiple subdomains and to the three-dimensional case in order to assess the potential of the method for complex geometries.

\section{Acknowledgements}

The authors greatly acknowledge the dissemination of his results and the fruitful discussion with Andreas Apostolatos from the Chair of Structural Analysis at Technische Universität München.

\section{References}

[1] Hughes TJR, Cottrell JA, Bazilevs Y. Isogeometric analysis: CAD, finite elements, NURBS, exact geometry and mesh refinement. Comput. Meth. Appl. Mech. Engrg. 2005; 194:4135-4195.

[2] Cottrell JA, Hughes TJR, Bazilevs Y. Isogeometric analysis: Toward integration of CAD and FEA. Wiley: Chichester, 2009.

[3] Piegl L, Tiller W. The NURBS book. second edn., Monographs in visual communications, Springer: Berlin, 1997.

[4] Bazilevs Y, Calo VM, Cottrell JA, Evans JA, Hughes TJR, Lipton S, Scott MA, Sederberg TW. Isogeometric analysis using T-splines. Comput. Meth. Appl. Mech. Engrg. 2010; 199:229-263.

[5] Cirak F, Scott MJ, Antonsson EK, Ortiz M, Schröder P. Integrated modeling, finite-element analysis and engineering design for thin-shell structures using subdivision. Comput. Aided Des. 2002; 34(2):137-148. 
[6] Wohlmuth BI. Discretization methods and iterative solvers based on domain decomposition, Lecture notes in computational science and engineering, vol. 17. Springer: Berlin and New York, 2001.

[7] Babuška I. The Finite Element Method with Penalty. Math. Comp. 1973; 27(122):221-228.

[8] Babuška I. The finite element method with Lagrangian multipliers. Numer. Math. 1973; 20:179-192.

[9] Nitsche J. Über ein Variationsprinzip zur Lösung von Dirichlet-Problemen bei Verwendung von Teilräumen, die keinen Randbedingungen unterworfen sind. Abh. Math. Semin. Univ. Hambg. 1971; 36(1):9-15.

[10] Bernardi C, Maday Y, Patera AT. Domain Decomposition by the Mortar Element Method. Asymptotic and Numerical Methods for Partial Differential Equations with Critical Parameters, Kaper HG, Garbey M, Pieper GW (eds.). Springer Netherlands: Dordrecht, 1993; $269-286$.

[11] Ben Belgacem F. The Mortar finite element method with Lagrange multipliers. Numer. Math. 1999; 84(2):173-197.

[12] Wohlmuth BI. A Mortar Finite Element Method Using Dual Spaces for the Lagrange Multiplier. SIAM J. Numer. Anal. 2000; 38(3):989-1012.

[13] Oswald P, Wohlmuth B. On polynominal reproduction of dual FE bases. Domain Decomposition Methods in Science and Engineering, 13th International Conference on Domain Decomposition Methods, Lyon, France, Debit N, Garbey M, Hoppe R, Keyes D, Kuznetsov Y, Périaux J (eds.). CIMNE, 2002; 85-96.

[14] Hesch C, Betsch P. Transient three-dimensional domain decomposition problems: Frame-indifferent mortar constraints and conserving integration. Int. J. Numer. Meth. Engng 2010; 82(3):329-358.

[15] Hesch C, Betsch P. Isogeometric analysis and domain decomposition methods. Comput. Meth. Appl. Mech. Engrg. 2012; 213-216:104-112.

[16] Brivadis E, Buffa A, Wohlmuth B, Wunderlich L. Isogeometric mortar methods. Comput. Meth. Appl. Mech. Engrg. 2015; 284:292-319.

[17] Apostolatos A, Schmidt R, Wüchner R, Bletzinger KU. A Nitsche-type formulation and comparison of the most common domain decomposition methods in isogeometric analysis. Int. J. Numer. Meth. Engng 2014; 97(7):473-504.

[18] Ruess M, Schillinger D, Özcan AI, Rank E. Weak coupling for isogeometric analysis of non-matching and trimmed multi-patch geometries. Comput. Meth. Appl. Mech. Engrg. 2014; 269:46-71.

[19] Nguyen VP, Kerfriden P, Brino M, Bordas SPA, Bonisoli E. Nitsche's method for two and three dimensional NURBS patch coupling. Comput. Mech. 2014; 53(6):1163-1182.

[20] Kagan P, Fischer A, Bar-Yoseph PZ. Mechanically based models: Adaptive refinement for B-spline finite element. Int. J. Numer. Meth. Engng. $2003 ; \mathbf{5 7}(8): 1145-1175$

[21] Cottrell JA, Hughes TJR, Reali A. Studies of refinement and continuity in isogeometric structural analysis. Comput. Meth. Appl. Mech. Engrg. 2007; 196:4160-4183.

[22] Benson DJ, Bazilevs Y, Hsu MC, Hughes TJR. Isogeometric shell analysis: The Reissner-Mindlin shell. Comput. Meth. Appl. Mech. Engrg. 2010; 199:276-289.

[23] Dornisch W, Klinkel S, Simeon B. Isogeometric Reissner-Mindlin shell analysis with exactly calculated director vectors. Comput. Meth. Appl. Mech. Engrg. 2013; 253:491-504.

[24] Dornisch W, Klinkel S. Treatment of Reissner-Mindlin shells with kinks without the need for drilling rotation stabilization in an isogeometric framework. Comput. Meth. Appl. Mech. Engrg. 2014; 276:35-66.

[25] Kiendl J, Bazilevs Y, Hsu MC, Wüchner R, Bletzinger KU. The bending strip method for isogeometric analysis of Kirchhoff-Love shell structures comprised of multiple patches. Comput. Meth. Appl. Mech. Engrg. 2010; 199:2403-2416.

[26] Dornisch W, Klinkel S. A new method for the connection of non-conforming NURBS patches in isogeometric analysis. Proc. Appl. Math. Mech. 2013; 13(1):117-118.

[27] Lawson CL, Hanson RJ. Solving least squares problems, Classics in applied mathematics, vol. 15. SIAM: Philadelphia, 1995.

[28] Wriggers P. Nonlinear finite element methods. Springer: Berlin and Heidelberg, 2008.

[29] Wagner W, Gruttmann F. A robust nonlinear mixed hybrid quadrilateral shell element. Int. J. Numer. Meth. Engng. 2005; 64(5):635-666. 